\title{
Peroxynitrite-Induced Neuronal Apoptosis Is Mediated by Intracellular Zinc Release and 12-Lipoxygenase Activation
}

\author{
Yumin Zhang, ${ }^{1}$ Hong Wang, ${ }^{1}$ Jianrong Li, ${ }^{1}$ Daniel A. Jimenez, ${ }^{2}$ Edwin S. Levitan, ${ }^{3}$ Elias Aizenman, ${ }^{2}$ and \\ Paul A. Rosenberg ${ }^{1}$ \\ 'Department of Neurology and Program in Neuroscience, Children's Hospital and Harvard Medical School, Boston, Massachusetts 02115, and Departments \\ of ${ }^{2}$ Neurobiology and ${ }^{3}$ Pharmacology, University of Pittsburgh School of Medicine, Pittsburgh, Pennsylvania 15261
}

\begin{abstract}
Peroxynitrite toxicity is a major cause of neuronal injury in stroke and neurodegenerative disorders. The mechanisms underlying the neurotoxicity induced by peroxynitrite are still unclear. In this study, we observed that TPEN $\left[N, N, N^{\prime}, N^{\prime}\right.$-tetrakis (2-pyridylmethyl)ethylenediamine], a zinc chelator, protected against neurotoxicity induced by exogenous as well as endogenous (coadministration of NMDA and a nitric oxide donor, diethylenetriamine NONOate) peroxynitrite. Two different approaches to detecting intracellular zinc release demonstrated the liberation of zinc from intracellular stores by peroxynitrite. In addition, we found that peroxynitrite toxicity was blocked by inhibitors of 12-lipoxygenase (12-LOX), p38 mitogen-activated protein kinase (MAPK), and caspase-3 and was associated with mitochondrial membrane depolarization. Inhibition of 12-LOX blocked the activation of p38 MAPK and caspase-3. Zinc itself induced the activation of 12-LOX, generation of reactive oxygen species (ROS), and activation of p38 MAPK and caspase-3. These data suggest a cell death pathway triggered by peroxynitrite in which intracellular zinc release leads to activation of 12-LOX, ROS accumulation, p38 activation, and caspase-3 activation. Therefore, therapies aimed at maintaining intracellular zinc homeostasis or blocking activation of 12-LOX may provide a novel avenue for the treatment of inflammation, stroke, and neurodegenerative diseases in which the formation of peroxynitrite is thought to be one of the important causes of cell death.
\end{abstract}

Key words: peroxynitrite; zinc; 12-lipoxygenase; caspase; reactive oxygen species; apoptosis

\section{Introduction}

Peroxynitrite-induced neurotoxicity is thought to be an important mechanism of neuronal injury in hypoxic-ischemic brain and spinal cord injury (Szabo, 1996) and a variety of neurodegenerative diseases, such as Alzheimer's disease and amyotrophic lateral sclerosis (ALS) (Chabrier et al., 1999; Heales et al., 1999). Peroxynitrite, a product formed in a near diffusion-limited reaction of nitric oxide and superoxide (Beckman and Koppenol, 1996), is a strong oxidizing agent that causes DNA damage (Szabo and Ohshima, 1997), lipid peroxidation (Radi et al., 1991), and protein nitration (Crow et al., 1997; Ara et al., 1998).

The mode of cell death induced by peroxynitrite can be necrosis, apoptosis, or mixed types of cell death, depending on peroxynitrite concentration, on the duration of peroxynitrite exposure, and on intracellular ATP levels (Bonfoco et al., 1995; Leist et al., 1997). Autophagy, an intracellular lysosome-mediated degradation of damaged or dysfunctional cytoplasmic components and intracellular organelles, may be another form of neuronal cell

Received Dec. 5, 2003; revised Sept. 10, 2004; accepted 0ct. 6, 2004.

This work was supported by grants from the Muscular Dystrophy Association, the United Cerebral Palsy Research Foundation, the Charitable Leadership Foundation, the Ron Shapiro Charitable Foundation, and National Institutes of Health Grants HD 18655, NS 38475, and NS 43277 (E.A.).

Correspondence should be addressed to Dr. Paul A. Rosenberg, Department of Neurology, Children's Hospital, 300 Longwood Avenue, Boston, MA 02115. E-mail: Paul.Rosenberg@tch.harvard.edu.

DOI:10.1523/JNEUROSCI.2469-04.2004

Copyright $\odot 2004$ Society for Neuroscience ～0270-6474/04/2410616-12\$15.00/0 death (Yuan et al., 2003). Activation of poly(ADP-ribose) polymerase by damaged DNA is suggested to be a major cause of peroxynitrite-induced necrosis (Eliasson et al., 1997; Szabo, 2003). However, the mechanism of peroxynitrite-induced apoptosis is less known. It has been reported that peroxynitrite can induce apoptosis in thymocytes (Virag et al., 1998), and the toxicity of thymocytes induced by peroxynitrite can be mediated by the release of zinc from intracellular stores (Virag and Szabo, 1999).

Zinc is the second most prevalent trace element in the body and is present in particularly high concentrations in the mammalian brain (Weiss et al., 2000). It is present in synaptic vesicles and is tightly bound to intracellular proteins and zinc fingercontaining transcription factors (Frederickson, 1989). The concentration of intracellular free zinc is thought to be very low under physiological conditions (Frederickson, 1989; Outten and O'Halloran, 2001). However, it can rise to $>300 \mathrm{~nm}$ in response to injurious stimuli (Canzoniero et al., 1999). The transsynaptic movement of zinc from presynaptic vesicles to postsynaptic neurons is thought to play a key role in neuronal injury after brain ischemia (Koh et al., 1996). Another important source of intracellular free zinc is derived from intracellular proteins or ligandbound zinc pools and contributes to the neurotoxicity induced by several oxidizing agents (Aizenman et al., 2000; McLaughlin et al., 2001; Du et al., 2002; Sensi et al., 2003). Whether peroxynitrite induces zinc release in neurons and the mechanism of zincinduced neurotoxicity are unclear, although energy depletion 
and reactive oxygen species (ROS) generation might be involved (Weiss et al., 2000; Dineley et al., 2003).

In this study, we aimed to determine whether peroxynitrite induced neurotoxicity is mediated through a pathway involving zinc release.

\section{Materials and Methods \\ Materials}

3-Morpholino-sydnonimine (SIN-1), peroxynitrite, and diethylenetriamine NONOate (DETA/NO) were obtained from Cayman Chemical (Ann Arbor, MI). Caspase inhibitors [Ac-Asp-Glu-Val-Asp-chloromethylketone (Ac-DEVD-CMK) and N-benzyloxycarbonyl-Val-Ala-Asp-fluoromethyl ketone (z-VAD-FMK)], caspase-3 substrate Ac-Asp-Glu-Val-Asp-7-amido4-(trifluoromethyl)coumarin (Ac-DEVD-AFC), and Mn (III)tetrakis (4benzoic acid) porphyrin chloride (MnTBAP) were obtained from Calbiochem (La Jolla, CA). Staurosporine was purchased from RBI (Natick, MA). 2,7-Dichlorofluorescein diacetate (Newport Green), FluoZin-3, dihydrorhodamine-123 (DHR-123), and 5,5',6,6' -tetrachloro-1,1',3,3'tetraethylbenzimidazolylcarbocyanine iodide (JC-1) were purchased from Molecular Probes (Eugene, OR). 2,3,5-Trimethyl-6-(12-hydroxy5-10-dodecadiynyl)-1,4-benzoquinone (AA861), 3-[1-( $p$-chlorobenzyl)-5-(isopropyl)-3-tert-butylthioindol]-2,2-dimethylpropanoic acid (MK-886), 7,7-dimethyleicosadienoic acid (DEDA), and indomethacin were purchased from Biomol (Plymouth Meeting, PA). BMD-122 (Nbenzyl- $N$-hydroxy-5-phenylpentanamide) was a gift from Dr. Lawrence J. Marnett (School of Medicine, Vanderbilt University, Nashville, TN). All other reagents were obtained from Sigma (St. Louis, MO).

\section{Tissue culture}

Neuronal cultures were prepared from embryonic day 16 Sprague Dawley rat fetuses using methods similar to those described previously (Dichter, 1978) but modified to produce cultures that contained $<1 \%$ astrocytes (Rosenberg and Aizenman, 1989; Rosenberg, 1991; Rosenberg et al., 1992; Wang et al., 1998). Cultures were initially plated on poly-Llysine-coated 24-well plastic plates (Costar, Cambridge, MA) using an 80:10:10 (v/v) mixture of DMEM (11960-010; Invitrogen, San Diego, $\mathrm{CA}$ ), Ham's F-12 (N-4888; Sigma), and heat-inactivated ironsupplemented calf serum (A2151; HyClone, Logan, UT) containing 2 mM glutamine, $25 \mathrm{~mm}$ HEPES, $24 \mathrm{U} / \mathrm{ml}$ penicillin, and $24 \mu \mathrm{g} / \mathrm{ml}$ streptomycin and maintained in a $5 \% \mathrm{CO}_{2}$ (balance air) incubator at $36^{\circ} \mathrm{C}$. Cell proliferation was inhibited by exposure to $5 \mu \mathrm{M}$ cytosine arabinoside at $24 \mathrm{hr}$ in vitro for $72 \mathrm{hr}$. On the fourth day of culture, the medium was removed completely and replaced with 90\% MEM, 10\% Nuserum IV (Collaborative Research, Bedford, MA), $2 \mathrm{~mm}$ glutamine, and $5 \mathrm{~mm}$ HEPES, containing $50 \mathrm{U} / \mathrm{ml}$ superoxide dismutase (Boehringer Mannheim, Indianapolis, IN), $20 \mathrm{U} / \mathrm{ml}$ catalase (CV-40; Sigma), $11 \mathrm{~mm}$ total glucose, and $9.3 \mathrm{~mm}$ total sodium bicarbonate, plus 2\% B27 supplement (17504-036; Invitrogen). Medium was not subsequently changed. To prevent evaporation of water, culture dishes were kept on "wet dishes" containing wet filter paper until they were used.

\section{Application of reagents}

In all experiments, 24-well culture plates were first washed two times in HBSS containing $0.1 \%$ bovine serum albumin (BSA) and then placed in Earle's balanced salt solution (EBSS), which is composed of the following (in $\mathrm{mm}$ ): $116 \mathrm{NaCl}, 5.4 \mathrm{KCl}, 1.8 \mathrm{CaCl}_{2}, 0.8 \mathrm{MgSO}_{4}, 26 \mathrm{NaHCO}_{3}, 1$ $\mathrm{NaH}_{2} \mathrm{PO}_{4}$, and $5.5 \mathrm{D}$-glucose. Experiments were performed exclusively in EBSS. All drugs were applied $15 \mathrm{~min}$ before the cells were exposed to SIN-1, peroxynitrite, NMDA, DETA/NO, or $\mathrm{ZnCl}_{2}$.

\section{Survival assay}

Neurons were exposed to SIN-1 ( $1 \mathrm{~mm}$ ) for $24 \mathrm{hr}$, and the morphological changes were examined by phase-contrast microscopy. Quantitation of survival of cells was determined $24 \mathrm{hr}$ after exposure to the test compounds by assessing the ability of cultures to reduce Alamar Blue (Trek Diagnostic Systems, Westlake, $\mathrm{OH}$ ) as an index of viability (McGahon et al., 1995). Details of this procedure have been provided previously (Back et al., 1998, 1999) and have been validated in examining the toxicity of SIN-1 in neurons (Zhang and Rosenberg, 2002). In brief, the test medium was removed after $24 \mathrm{hr}$ and then replaced with EBSS plus a 1:100 dilution of Alamar Blue. After $2 \mathrm{hr}$ of exposure, $100 \mu \mathrm{l}$ of medium from each well (24-well plate) was transferred to a 96-well plate, and the fluorescence of the Alarmar Blue solution in each well was read in a fluorescence plate reader (FluoroCount; Packard, Meriden, CT), with excitation set at $\lambda=530 \mathrm{~nm}$ and emission set at $\lambda=590 \mathrm{~nm}$ at room temperature (RT).

\section{Fluorescence imaging of intracellular liberation of zinc}

Assay of direct interaction of peroxynitrite with fluorescent $\mathrm{Zn}^{2+}$ indicators. The reaction of peroxynitrite with the $\mathrm{Zn}^{2+}$ fluorescent indicators Newport Green and FluoZin-3 was examined in a cell-free system. The hydrolysis of Newport Green and FluoZin-3 AM was performed following the method described by LeBel et al. (1992). Newport Green (1 mM) or FluoZin-3 ( $1 \mathrm{~mm}$ ) was dissolved in $50 \mu \mathrm{l}$ of DMSO, and then an equal volume of methanol was added. After deesterification with $0.4 \mathrm{ml}$ of $\mathrm{NaOH}(10 \mathrm{~mm}), 2 \mathrm{ml}$ of $\mathrm{NaH}_{2} \mathrm{PO}_{4}(25 \mathrm{~mm}$; pH 7.4) was added for neutralization. The hydrolyzed $\mathrm{Zn}^{2+}$ indicator was diluted 1:100 into EBSS. After addition of peroxynitrite $(100 \mu \mathrm{M})$, the intensity of fluorescence was measured by a Hitachi (Tokyo, Japan) fluorescence spectrophotometer with an excitation wavelength at $485 \mathrm{~nm}$ and an emission wavelength at $530 \mathrm{~nm}$.

Assay of intracellular liberation of zinc using FluoZin-3. Changes in intracellular free $\mathrm{Zn}^{2+}$ concentration in neurons were monitored with a high-affinity, zinc-selective indicator, FluoZin-3 (Gee et al., 2002). Neurons were loaded with FluoZin-3 $(1 \mu \mathrm{M})$ for $30 \mathrm{~min}$ and then washed twice with HBSS containing $0.1 \%$ BSA. At $30 \mathrm{~min}$ after neurons were treated with peroxynitrite $(100 \mu \mathrm{M})$, fluorescence imaging of intracellular zinc was monitored immediately using digital fluorescence microscopy with a $20 \times$ objective (excitation at $485 \mathrm{~nm}$; emission at $530 \mathrm{~nm}$ ). For all images, the microscope settings, such as brightness, contrast, and exposure time, were held constant to compare the relative intensity of intracellular zinc fluorescence across all treatment conditions.

Assay of intracellular liberation of zinc by fluorescence resonance energy transfer. Neurons were transfected using Lipofectamine 2000 (Invitrogen) (Pal et al., 2003), with the cDNA of the modified cameleon-2 probe that contains the human metallothionein (MT) IIa cDNA flanked by the cDNA of two mutant green fluorescent proteins, enhanced cyan fluorescent protein (ECFP) and enhanced yellow fluorescent protein (EYFP) (Pearce et al., 2000). In brief, $1.5 \mu \mathrm{g}$ of cDNAs was diluted in $50 \mu \mathrm{l}$ of Opti-Mem I medium and combined with $50 \mu \mathrm{l}$ of Opti-Mem I medium containing $4 \mu \mathrm{l}$ of Lipofectamine 2000. Complexes were allowed to form for $30 \mathrm{~min}$ at RT before addition to the cultures. Cells were maintained for $24-48 \mathrm{hr}$ at $37^{\circ} \mathrm{C}, 5 \% \mathrm{CO}_{2}$ before imaging. Dual emission imaging was accomplished by using a 440 DF20 excitation filter, a 455 DRLP dichroic mirror, and alternating emission filters (480DF30 for ECFP; 535DF25 for EYFP). With the cameleon-2 construct, it has been shown that an increase in emission signal at $480 \mathrm{~nm}$ that is accompanied by a decrease in emission at $535 \mathrm{~nm}$ is reflective of a loss of bound $\mathrm{Zn}^{2+}$ ions by the metallothionein construct [i.e., less fluorescence resonance energy transfer (FRET)] (Pearce et al., 2000). Positively transfected cells were monitored for changes in the 535/480 emission intensity ratio after exposure to $\mathrm{Zn}^{2+}$ plus pyrithione or the thiol oxidant 2,2'dithiodipyridine (DTDP), in addition to peroxynitrite. DTDP is an agent that we have shown previously is able to liberate intracellular $\mathrm{Zn}^{2+}$ from neurons (Aizenman et al., 2000) and can liberate all seven $\mathrm{Zn}^{2+}$ atoms from metallothionein in cell-free assays (Maret and Vallee, 1998).

\section{Assay of intracellular ATP}

The Bioluminescent Somatic Cell Assay kit from Sigma (catalog \#FLASC) was used for assay of ATP, as described previously (Rosenberg et al., 2000; Zhang and Rosenberg, 2002). At selected time points after addition of drug or vehicle, medium was removed and then replaced with $200 \mu \mathrm{l}$ of cell lysis agent. After brief agitation, the medium was transferred to microcentrifuge tubes that contained $200 \mu \mathrm{l}$ of water. The tubes were placed on wet ice until assay. ATP assay mix was used at 25 -fold dilution, and $100 \mu \mathrm{l}$ of the extract was assayed. The proteins in cell lysates were quantified using a protein assay kit (Bio-Rad, Hercules, CA). 


\section{Gluthathione measurement}

Intracellular levels of glutathione were assayed using monochlorobimane (MCB), as described previously (Wang and Joseph, 2000). MCB reacts with gluthathione (GSH) in a reaction catalyzed by glutathione $S$-transferase (GST) to form a highly fluorescent adduct. Briefly, cultures were exposed to SIN-1 (1 mM) for $30 \mathrm{~min}$ and then washed twice with HBSS containing $0.1 \%$ BSA. Neurons were then lysed with $0.2 \%$ Triton X-100 in EBSS. In $100 \mu$ l cell lysates, MCB $(50 \mu \mathrm{M})$ and GST $(0.4 \mathrm{U} / \mathrm{ml})$ were added and incubated at $37^{\circ} \mathrm{C}$ for $20 \mathrm{~min}$. Fluorescence was measured with the excitation wavelength at $360 \mathrm{~nm}$ and emission wavelength at $480 \mathrm{~nm}$. The concentration of GSH was calculated based on the standard curve of GSH and on the protein concentration of the sample.

\section{Assay of 12-lipoxygenase activity}

12-Lipoxygenase (12-LOX) activity was measured by quantifying the major metabolic product of 12-LOX, 12-hydroxyeicosatetraenoic acid (12-HETE), by using an ELISA kit (Assay Designs, Ann Arbor, MI). Neurons were treated with SIN-1 or $\mathrm{ZnCl}_{2}$ for $60 \mathrm{~min}$. After the cultures were washed twice with HBSS containing $0.1 \mathrm{mg} / \mathrm{ml} \mathrm{BSA}$, arachidonic acid $(\mathrm{AA} ; 20 \mu \mathrm{M})$ in EBSS was added to the culture, which was then incubated for $30 \mathrm{~min}$. The medium was then collected, extracted, and assayed for 12-HETE according to the manufacturer's protocol. The concentration of 12-HETE in each sample was normalized to the protein concentration of the sample.

\section{Measurement of intracellular free-radical generation}

Intracellular free-radical generation was evaluated with dihydrorhodamine 123 (Rho123) (Royall and Ischiropoulos, 1993). At $2 \mathrm{hr}$ after zinc exposure (90 min), cells were loaded with Rho123 (10 $\mu \mathrm{M})$ for $20 \mathrm{~min}$ at $37^{\circ} \mathrm{C}$ and then washed once with EBSS. Fluorescent imaging of oxidized Rho123 was performed immediately using digital fluorescence microscopy with a $20 \times$ objective (excitation at $490 \mathrm{~nm}$ and emission at $515 \mathrm{~nm}$ ). All of the images were taken with the same microscope settings.

\section{Assay of the mitochondrial membrane potential}

JC-1 (Molecular Probes) was used as an indicator for mitochondrial membrane potential $(\Psi \mathrm{m})$, as described previously (Salvioli et al., 1997). This carbocyanine exists in a monomeric form in the cytosol, emitting at $527 \mathrm{~nm}$ (green) when $\Psi \mathrm{m}$ is low. When JC-1 is concentrated by actively respiring mitochondria, it forms aggregates that are associated with a large shift in emission (590 nm) (red). A final concentration of $1 \mu \mathrm{g} / \mathrm{ml}$ JC- 1 was added directly into the culture at $5 \mathrm{hr}$ after $90 \mathrm{~min}$ of SIN-1 treatment. For the drug-treated groups, drugs were added during and after 90 min of SIN-1 exposure. At 20 min after the loading with JC-1, cells were visualized using digital fluorescence microscopy with a $20 \times$ objective. The green fluorescence and red fluorescence were detected using two optical filters designed for fluorescein and tetramethylrhodamine, respectively. Quantitation of $\Psi \mathrm{m}$ changes in neurons treated with SIN-1 was also performed, using a fluorescent plate reader. The green fluorescence of monomeric JC-1 was read with excitation wavelength at $485 \mathrm{~nm}$ and emission wavelength at $530 \mathrm{~nm}$. The red fluorescence of the aggregated JC- 1 was read with excitation wavelength at 485 $\mathrm{nm}$ and emission wavelength at $590 \mathrm{~nm}$. The percentage decrease in the 590/530 emission intensity ratio in SIN-1-treated neurons compared with control neurons reflects the loss of $\Psi \mathrm{m}$.

\section{Assay of caspase- 3 activation}

The activation of caspase- 3 was examined using a caspase- 3 inhibitor (DEVD-FMK) conjugated to FITC as the fluorescent in situ marker in living cells (catalog \#QIA91; Oncogene, San Diego, CA). FITC-DEVDFMK is cell permeable, nontoxic, and irreversibly binds to activated caspase-3 in apoptotic cells. At $7 \mathrm{hr}$ after exposure of neurons to SIN-1 (1 $\mathrm{mm}$ for $90 \mathrm{~min}$ ) or zinc (75 $\mu \mathrm{M}$ for $90 \mathrm{~min}$ ) or at $5 \mathrm{hr}$ after staurosporine (STS) $(1 \mu \mathrm{M})$ exposure, the cultures were washed twice with HBSS containing $0.1 \%$ BSA and then incubated with FITC-DEVD-FMK $(1 \mu \mathrm{l} / \mathrm{ml}$ in EBSS) for $1 \mathrm{hr}$ in a $37^{\circ} \mathrm{C}$ incubator with $5 \% \mathrm{CO}_{2}$. The FITC label in apoptotic cells was examined immediately under fluorescence microcopy with excitation and emission at 485 and $535 \mathrm{~nm}$, respectively. All of the images were taken with the same microscope settings.

The activity of caspase- 3 was measured following the methods de- scribed by Stefanis et al. (1999). At $7 \mathrm{hr}$ after exposure of neurons to SIN-1 ( $1 \mathrm{~mm}$ for $90 \mathrm{~min}$ ) or at $5 \mathrm{hr}$ after STS $(1 \mu \mathrm{M})$ exposure, the cultures were washed twice with HBSS containing $0.1 \%$ BSA and then lysed with a buffer containing the following (in mM): 25 HEPES, pH 7.5, 5 EDTA, 1 EGTA, $5 \mathrm{MgCl}_{2}, 2 \mathrm{DTT}$, and a mixture of protease inhibitors (catalog \#1836153; Roche, Indianapolis, IN). The cell lysates were put on ice for $20 \mathrm{~min}$ and then sonicated on ice. The lysates were centrifuged for $60 \mathrm{~min}$ at $16,000 \times g$. The protein concentrations in the supernatant were determined using a protein assay kit (Bio-Rad). Ten micrograms of protein were incubated in a buffer of 25 mM HEPES, pH 7.5, $10 \%$ sucrose, $0.1 \%$ 3-[(3-cholamidopropyl) dimethylammonio]-1-propanesulfonic acid, and $10 \mathrm{~mm}$ DTT with the caspase-3 substrate Ac-DEVD-AFC $(15 \mu \mathrm{M})$ at $37^{\circ} \mathrm{C}$ for $1 \mathrm{hr}$. The intensity of fluorescence of each solution was measured by a Hitachi fluorescence spectrophotometer using an excitation wavelength of $400 \mathrm{~nm}$ and an emission wavelength of $505 \mathrm{~nm}$.

\section{Western blot analysis of $p 38$ activation}

At $1 \mathrm{hr}$ after SIN-1 treatment, neurons were placed on ice. After media aspiration, cells were washed once with ice-cold PBS and lysed with lysis buffer containing $20 \mathrm{~mm}$ Tris, pH 7.5, $150 \mathrm{~mm} \mathrm{NaCl}, 1 \mathrm{~mm}$ EDTA, $1 \mathrm{~mm}$ EGTA, 1\% Triton X-100, 2.5 mm sodium pyrophosphate, 1 mm glycerolphosphate, $1 \mathrm{~mm} \mathrm{Na}_{3} \mathrm{VO}_{4}$, and $1 \mathrm{~mm}$ PMSF. An aliquot of cell lysate was removed for later protein determination. Cell lysate was mixed with Laemmli buffer, boiled for $5 \mathrm{~min}$, and stored at $-20^{\circ} \mathrm{C}$. Equal amounts of proteins were separated by SDS-PAGE and electrotransfered to polyvinylidene difluoride membrane. The membrane was blocked with $5 \%$ nonfat milk in TBS containing $0.1 \%$ Tween 20 (TBST) for $1 \mathrm{hr}$ and then incubated overnight at $4^{\circ} \mathrm{C}$ with the primary antibody for phosphorylated p38 (Biosource, Camarillo, CA) or total p38 (Cell Signaling, Beverly, MA) diluted at 1:1000 in TBST containing 5\% BSA. After washing four times with TBST, the membrane was incubated for $1 \mathrm{hr}$ at RT with an HRP-conjugated anti-rabbit secondary antibody (Santa Cruz Biotechnology, Santa Cruz, CA) diluted 1:4000. Membranes were washed again as above and visualized by ECL according to the manufacturer's protocol.

\section{Statistics}

Statistical significance was assessed using ANOVA with the TukeyKramer post hoc multiple comparison test. Statistical analysis was performed using the Instat program from GraphPad Software (San Diego, CA). Representative experiments are shown unless noted otherwise. Experiments were performed with triplicate samples, and the data are expressed as mean $\pm \mathrm{SD}$ or SEM, as appropriate. All experiments were repeated at least three times.

\section{Results}

$N, N, N^{\prime}, N^{\prime}$-tetrakis (2-pyridyl-methyl)ethylenediamine, a zinc chelator, protected against neurotoxicity induced by peroxynitrite

SIN-1 is a commonly used peroxynitrite generator, because it can simultaneously generate nitric oxide and superoxide in aerobic conditions (Hogg et al., 1992). Administration of SIN-1 (1 mM) to rat forebrain neurons in culture caused severe neurotoxicity (Fig. 1A). In five experiments, the neuronal survival after $24 \mathrm{hr}$ was $10 \pm 5.5 \%$ of control levels. $N, N, N^{\prime}, N^{\prime}$-tetrakis (2-pyridylmethyl)ethylenediamine (TPEN), a zinc chelator that has been shown to protect against peroxynitrite-induced toxicity in thymocytes (Virag and Szabo, 1999), was able to attenuate SIN-1induced toxicity to neurons (Fig. $1 A$ ). Neuronal survival in the presence of TPEN $(1 \mu \mathrm{M})$ was $68 \pm 5 \%(n=5)$, which is significantly higher $(p<0.001)$ than in the SIN-1-treated group. TPEN also blocked the neurotoxicity induced by authentic peroxynitrite $(100 \mu \mathrm{M})$ (Fig. $1 \mathrm{~B})$. The $\mathrm{EC}_{50}$ value of TPEN protection was $0.95 \pm 0.03 \mu \mathrm{M}(n=3)$. To test the pathophysiological relevance of the protective effect of TPEN against peroxynitrite, we used a model system in which the formation of peroxynitrite might occur in neurons as a result of reaction of exogenous nitric 

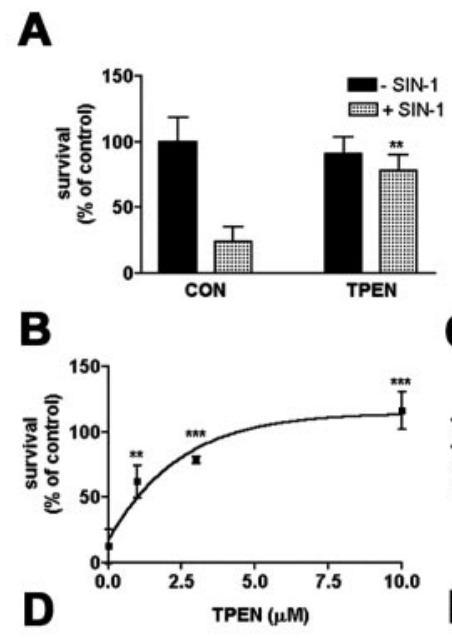

\section{C}
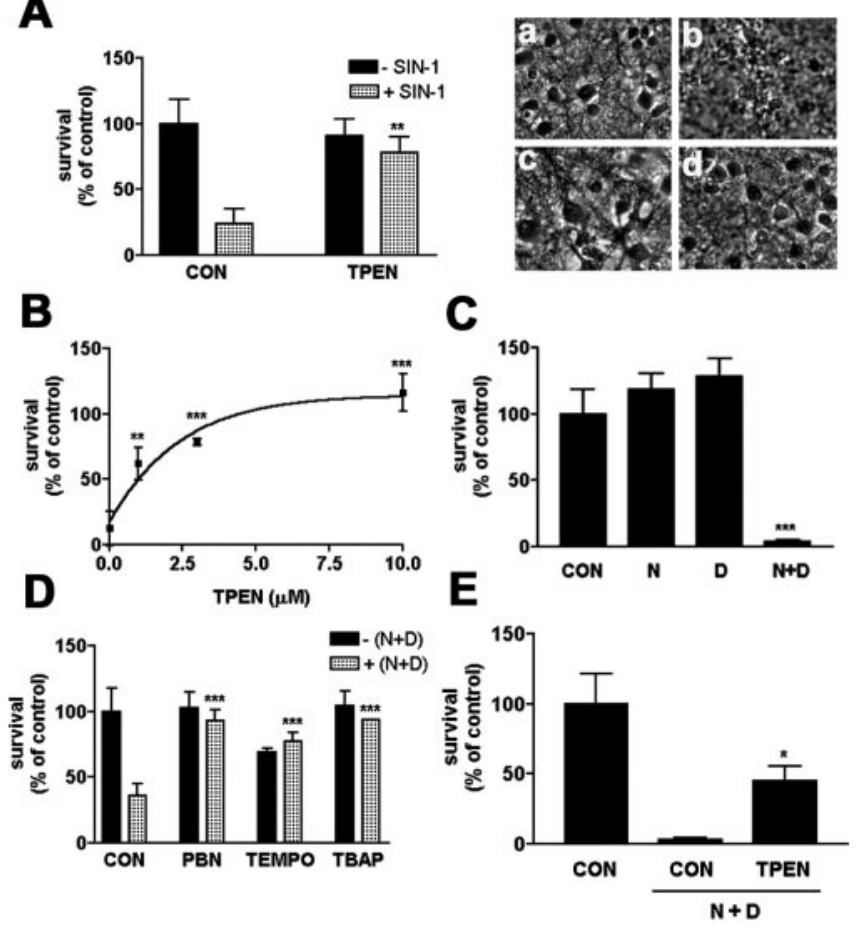

Figure 1. TPEN is protective against neurotoxicity induced by peroxynitrite. A, TPEN protected against SIN-1-induced neurotoxicity. Neurons were exposed to SIN-1 (1 mM) with and without TPEN (1 $\mu \mathrm{m})$ for $24 \mathrm{hr}$, and then the neurotoxicity was examined. ${ }^{* *} p<0.01$ was obtained when the TPEN-treated group was compared with the group treated with SIN-1 alone. $a$, Control; $b$, SIN-1; $c$, TPEN; $d$, TPEN/SIN-1. One representative experiment of five performed is shown. $B$, TPEN protected against neurotoxicity induced by authentic peroxynitrite. Neurons were exposed to authentic peroxynitrite $(100 \mu \mathrm{M})$ in the absence or presence of various concentrations of TPEN $(1,3,10 \mu \mathrm{m})$ for $24 \mathrm{hr}$, and then the neurotoxicity was examined. ${ }^{* *} p<$ 0.01 and ${ }^{* * *} p<0.001$ were obtained when the TPEN-treated groups were compared with the group treated with peroxynitrite alone. One representative experiment of three performed is shown. C, Nitric oxide has a synergistic effect with NMDA in producing neurotoxicity. Neurons were exposed to NMDA (N) $(20 \mu \mathrm{M})$, DETA/NO (D) $(300 \mu \mathrm{m})$, or NMDA plus DETA/NO (N+D) for $24 \mathrm{hr}$, and then the neurotoxicity was examined. ${ }^{* * *} p<0.001$ was obtained when the NMDA plus DETA/NO-treated group was compared with other groups. One representative experiment of six performed is shown. $D$, Free-radical spin traps blocked neurotoxicity induced by NMDA plus DETA/NO. Neurons were exposed to NMDA $(20 \mu \mathrm{m})$ plus DETA/NO $(300 \mu \mathrm{m})$ in the absence or presence of free-radical spin traps (10 mм PBN or $1 \mathrm{~mm}$ TEMP0) or MnTBAP $(100 \mu \mathrm{m})$ for $4 \mathrm{hr}$, and then the neurotoxicity was examined. ${ }^{* * *} p<0.001$ was obtained when the drug-treated groups were compared with the group treated with NMDA plus the DETA/N0-treated group alone. One representative experiment of four performed is shown. E, TPEN protected against neurotoxicity induced by NMDA plus DETA/NO. Neurons were exposed to NMDA (20 $\mu \mathrm{m})$ plus DETA/NO $(300 \mu \mathrm{m})$ for $1 \mathrm{hr}$; the toxicity was examined at $24 \mathrm{hr} .{ }^{*} p<0.05$ was obtained when the TPEN-treated group was compared with the group treated with NMDA plus DETA/NO alone. One representative experiment of three performed is shown. CON, Control. Error bars represent SD.

oxide with superoxide generated intracellularly in response to an excitotoxic stimulus (Hewett et al., 1994). It has been shown previously that exposure of neurons to NMDA can generate superoxide (Hewett et al., 1994; Patel et al., 1996; Sengpiel et al., 1998). Therefore, when a nitric oxide donor is applied to the cultures treated with NMDA, nitric oxide may react with superoxide to form peroxynitrite intracellularly. We used conditions of exposure to NMDA ( $24 \mathrm{hr} ; 20 \mu \mathrm{M})$ or the nitric oxide donor DETA/NO (24 hr; $300 \mu \mathrm{M})$ that did not, by themselves, result in toxicity. However, when NMDA and DETA/NO were coapplied, a nearly complete loss of neurons was observed (Fig. 1C). We found that the free-radical spin traps [N-t-butyl- $\alpha$ phenylnitrone $(\mathrm{PBN})$ and 2,2,6,6-tetramethylpiperidine 1-oxyl (TEMPO)] and MnTBAP, a scavenger of superoxide as well as

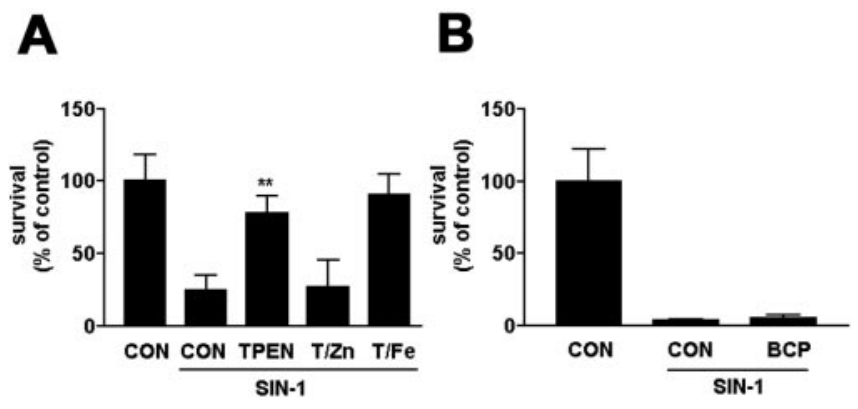

Figure 2. Peroxynitrite-induced neurotoxicity is mediated by zinc but not by iron or copper. $A$, TPEN $(1 \mu \mathrm{m})$ protection against SIN-1-induced neurotoxicity was eliminated by coadministration of an equimolar concentration of $\mathrm{ZnCl}_{2}(\mathrm{~T} / \mathrm{Zn})(1 \mu \mathrm{m})$ but not of $\mathrm{FeCl}_{2}(\mathrm{~T} / \mathrm{Fe})(1 \mu \mathrm{m})$. ${ }^{* *} p<0.01$ was obtained when the TPEN-treated group was compared with the group treated with SIN-1 alone. One representative experiment of three performed is shown. B, Copper chelator had no effect on SIN-1-induced neurotoxicity. $B C P(100 \mu \mathrm{m})$ represents bathocuproine. One representative experiment of three performed is shown. CON, Control. Error bars represent SD.

peroxynitrite, completely blocked neurotoxicity induced by a 4 hr exposure to NMDA plus DETA/NO (Fig. 1D). The toxicity induced by NMDA and DETA/NO was also significantly attenuated by TPEN at $10 \mu \mathrm{M}$ (Fig. $1 E$ ).

TPEN is not only a chelator of zinc, it may also chelate other divalent cations, with high affinity for copper and iron and low affinity for calcium and magnesium (Jornot et al., 1998; Virag and Szabo, 1999; Armstrong et al., 2001). An equimolar concentration of $\mathrm{ZnCl}_{2}(1 \mu \mathrm{M})$ completely eliminated the neuroprotection of TPEN (1 $\mu \mathrm{M})$ against SIN-1 (1 mM) toxicity (Fig. 2A). However, an equimolar concentration of $\mathrm{FeCl}_{2}$ had no effect on the protective effect of TPEN (Fig. 2A). Because TPEN has a higher affinity for copper than zinc $\left(K_{\mathrm{D}}, 10^{-20} \mathrm{M}\right.$ for $\mathrm{Cu}^{2+}$ and $10^{-15} \mathrm{M}$ for $\mathrm{Zn}^{2+}$ ) (McCabe et al., 1993), we examined the effect of a specific copper chelator on the toxicity induced by peroxynitrite. Bathocuprione at $100 \mu \mathrm{M}$ (Armstrong et al., 2001) was not protective against SIN-1 (Fig. $2 B$ ), authentic peroxynitrite, or NMDA plus DETA/NO (data not shown). It is possible that the failure of bathocuprone to protect against peroxynitrite-induced toxicity is attributable to its poor ability to cross membranes. To exclude this possibility, we compared the effect of bathocuprone with a known extracellular metal chelator, EDTA, on neurotoxicity induced by SIN-1. EDTA at $500 \mu \mathrm{M}$ completely blocked SIN-1 toxicity, but bathocuprone at $500 \mu \mathrm{M}$ had no effect (data not shown). If the protective effect of EDTA was attributable to its chelation of copper, then bathocuprone, a specific copper chelator, should also be protective, regardless of whether it gets into neurons. These results suggested that copper does not mediate peroxynitrite-induced neurotoxicity. EBSS contains a much higher concentration of calcium $(1.8 \mathrm{~mm})$ and magnesium $(0.8 \mathrm{mM})$ than the concentration of TPEN $(1 \mu \mathrm{M})$ used in these experiments. Therefore, the protective effect of TPEN is probably not attributable to the chelation of calcium or magnesium. These results suggested that TPEN exerts its protective effect by chelating zinc released from intracellular stores.

\section{Peroxynitrite induced intracellular free zinc accumulation}

Newport Green and FluoZin-3 have been used as fluorescent $\mathrm{Zn}^{2+}$ indicators to monitor the intracellular free $\mathrm{Zn}^{2+}$ concentrations under oxidative stress (Aizenman et al., 2000; Gee et al., 2002). To make sure that these indicators do not directly react with peroxynitrite, a strong oxidative agent, we first tested them in the presence of peroxynitrite $(100 \mu \mathrm{M})$ in EBSS without cells present. Peroxynitrite caused an increase in the fluorescence of 
Newport Green independent of the $\mathrm{Zn}^{2+}$ levels (Fig. 3A). No reaction occurred between peroxynitrite and FluoZin-3 (Fig. $3 B$ ). Therefore, FluoZin-3 was used to examine whether peroxynitrite could induce zinc release from neurons.

Neurons were preloaded with FluoZin-3 $(1 \mu \mathrm{M})$ for $30 \mathrm{~min}$, washed twice with HBSS containing $0.1 \% \mathrm{BSA}$, and then treated with peroxynitrite $(100 \mu \mathrm{M})$ for $30 \mathrm{~min}$ in the absence or presence of TPEN $(10 \mu \mathrm{M})$. Peroxynitrite induced an increase in zinc fluorescence in neurons, and the effect was completely diminished by coapplication of TPEN (Fig. 3C).

We also assayed for intracellular zinc release induced by peroxynitrite using intramolecular FRET. Thiol oxidants and nitric oxide have been shown to induce the release of $\mathrm{Zn}^{2+}$ from MTs in a variety of experimental paradigms (Kroncke et al., 1994; Berendji et al., 1997; Aravindakumar et al., 1999; Aizenman et al., 2000; Pearce et al., 2000; Bossy-Wetzel et al., 2004; Pal et al., 2004). Pearce et al. (2000) described a modification of a chimeric "cameleon" probe (Miyawaki et al., 1997), with which conformational changes in MT, resulting from metal binding and unbinding, could be detected by FRET. We used this probe in transfected neurons to determine whether peroxynitrite could induce the release of $\mathrm{Zn}^{2+}$ from metallothionein. Because this probe had not been used previously in neurons, we first characterized its behavior in these cells. We reasoned that as a result of the overexpression of MT-FRET in transfected neurons, a substantial portion of the expressed protein would be in the apo or thionein form and that addition of $\mathrm{Zn}^{2+}$ would result in an increase in FRET (i.e., an increase in the 535/480 emission intensity ratio resulting from the binding of $\mathrm{Zn}^{2+}$; see Materials and Methods). Indeed, this was exactly what was observed after $\mathrm{Zn}^{2+}$ exposure (Fig. 3D). Conversely, FRET was readily decreased (i.e., the 535/480 emission ratio decreased after $\mathrm{Zn}^{2+}$ unbinding) after exposure to the thiol-oxidizing agent DTDP, as expected from previous studies (Maret and Vallee, 1998; Aizenman et al., 2000) (Fig. 3E). From these data, it appeared that the MT-FRET protein was behaving as predicted (i.e., on binding zinc, FRET increased, whereas releasing zinc resulted in a decrease in FRET). We thus were in a position to be able to test whether an application of peroxynitrite would result in a decrease in FRET, reflective of $\mathrm{Zn}^{2+}$ liberation from the MT-containing chimeric indicator. We observed that addition of freshly prepared peroxynitrite (140 $\mu \mathrm{M})$ produced a relatively slow, albeit reproducible and pronounced decline in the 535/480 emission ratio (Fig. $3 F$ ). The time course of the change in FRET is likely attributable to the fact that peroxynitrite is known to interact relatively slowly with most biological molecules (Beckman and Koppenol, 1996). After removal of peroxynitrite, the FRET signal continued to decline for
D
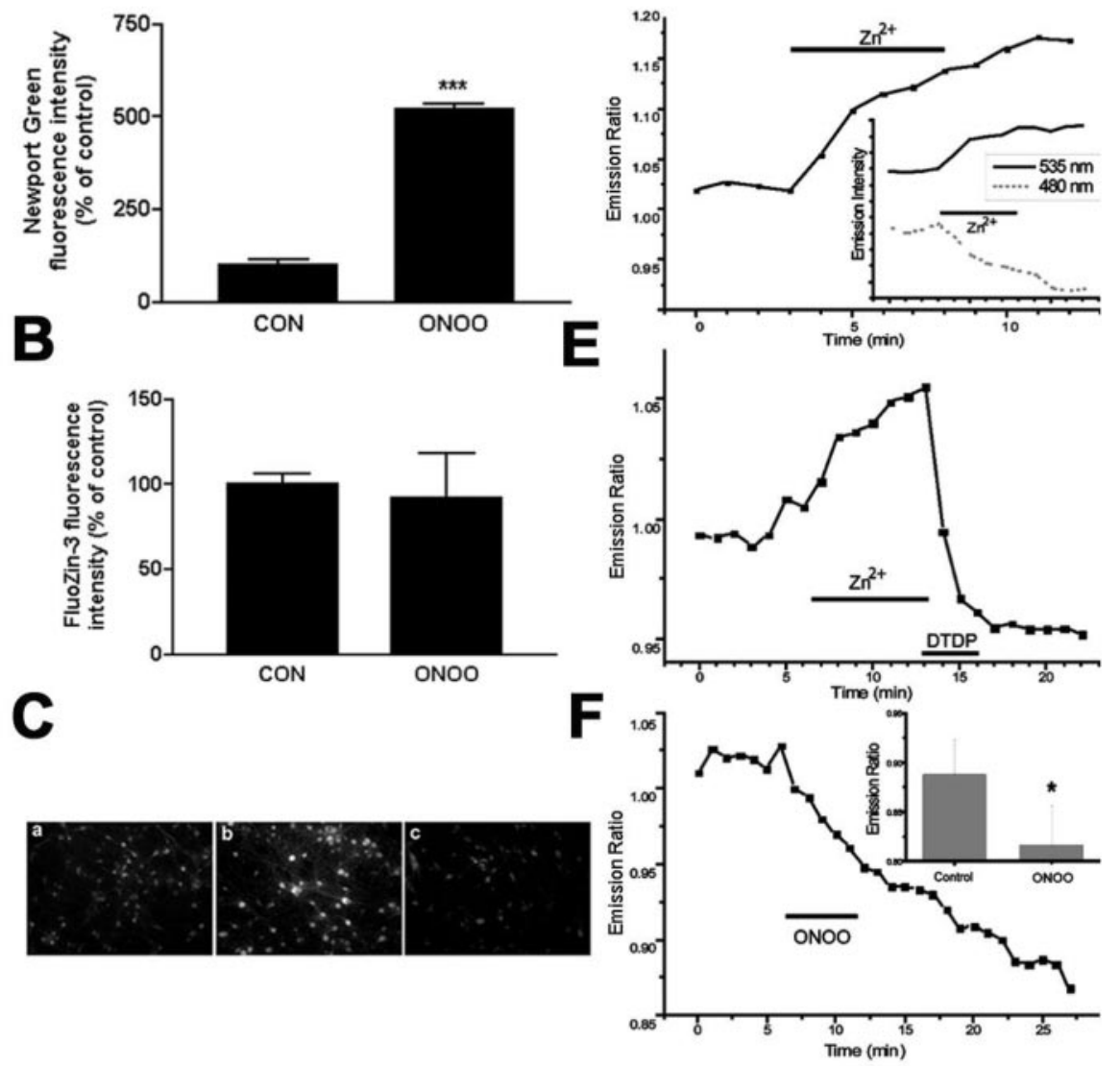

Figure 3. Peroxynitrite induced intracellular free zinc accumulation. $A, B$, Interaction of peroxynitrite with the fluorescent zinc indicators Newport Green and FluoZin-3. A, Peroxynitrite (ONOO) induced an increase in Newport Green fluorescence in EBSS. ${ }^{* * *} p<0.001$ when compared with the control (CON). B, Peroxynitrite did not interact with FluoZin-3. The fluorescence intensity 作 induced by peroxynitrite. Neurons were preloaded with FluoZin- 3 for $30 \mathrm{~min}$, washed, and then treated with peroxynitrite in the ECFP and EYFP. Twenty-four hours after transfection, cells $(n=52)$ were monitored for changes in the $535 / 480$ emission intensity

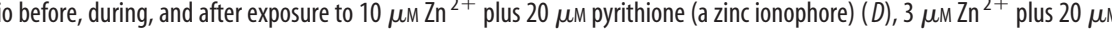
$\mathrm{Zn}^{2+}$ to protein not saturated by the metal, whereas a decrease in the ratio indicates the liberation of $\mathrm{Zn}^{2+}$ from MT-FRET. Inset the $535 / 480$ ratio (mean $\pm \mathrm{SEM} ; n=7$ ) immediately before (control) and 5 min after the peroxynitrite treatment $\left({ }^{*} p<0.001\right.$; two-tailed paired $t$ test). Error bars represent SD.

many minutes, suggesting that longer-lived peroxynitrite oxidation products (Beckman and Koppenol, 1996) may contribute to MT oxidation and $\mathrm{Zn}^{2+}$ liberation.

\section{TPEN had no effect on peroxynitrite-induced ATP depletion}

An increasing body of evidence suggests that zinc can cause inhibition of cellular energy production (Dineley et al., 2003). Sheline et al. (2000) showed that elevation of extracellular zinc could inhibit glycolysis in cultured neurons and that pyruvate, a downstream substrate of glycolysis, had a protective effect. Here, we tested whether pyruvate could block ATP depletion and neurotoxicity induced by peroxynitrite. Pyruvate at $10 \mathrm{~mm}$ did not block neurotoxicity induced by $24 \mathrm{hr}$ of SIN-1 exposure (Fig. $4 A$ ). However, when neurotoxicity was assessed at $24 \mathrm{hr}$ after a short period (1-3 hr) of SIN-1 exposure, pyruvate had a significant protective effect (Fig. 4B). Pyruvate also blocked ATP deple- 

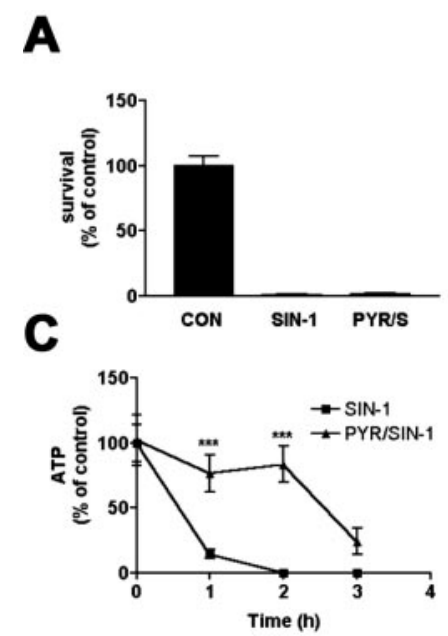

B
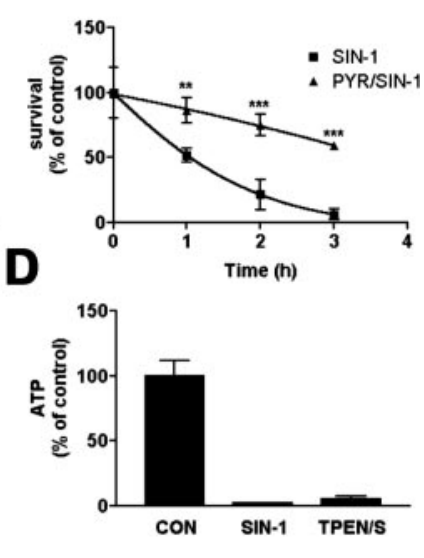

Figure 4. Effect of pyruvate on SIN-1-induced ATP depletion and neurotoxicity. A, Pyruvate did not prevent neurotoxicity induced by $24 \mathrm{hr}$ of SIN-1 exposure. Neurons were exposed to SIN-1 (1 mm) in the absence or presence of pyruvate $(10 \mathrm{~mm})$ for $24 \mathrm{hr}$, and then the toxicity was examined. PYR/S, Pyruvate plus SIN-1. One representative experiment of three performed is shown. $B$, Pyruvate (PYR; $10 \mathrm{~mm}$ ) protected against neurotoxicity at $24 \mathrm{hr}$ after $1-3 \mathrm{hr}$ of SIN-1 exposure. ${ }^{* *} p<0.01$ and ${ }^{* * *} p<0.001$ were obtained when pyruvate-treated groups were compared with the corresponding groups treated with SIN-1 alone. One representative experiment of three performed is shown. C, Pyruvate $(10 \mathrm{~mm})$ blocked ATP depletion induced by 1-2 hr of SIN-1 (1 mm) exposure but not by $3 \mathrm{hr}$ of SIN-1 exposure. The cellular levels of ATP were examined in neurons treated with SIN-1 or SIN-1 together with pyruvate at 1, 2, and $3 \mathrm{hr}$. ${ }^{* * *} p<0.001$ was obtained when pyruvate-treated groups were compared with the corresponding groups treated with SIN-1 alone. One representative experiment of three performed is shown. D, TPEN did not block ATP depletion induced by SIN-1. Neurons were exposed to SIN-1 with or without TPEN for $1 \mathrm{hr}$, and then the cellular levels of ATP were examined. One representative experiment of three performed is shown. CON, Control; TPEN/S, TPEN plus SIN-1. Error bars represent SD.

tion after 1-2 hr of SIN-1 exposure but failed to block ATP depletion when neurons were exposed to SIN-1 for $3 \mathrm{hr}$ (Fig. 4C), although significant neuroprotection was observed at $24 \mathrm{hr}$ after $3 \mathrm{hr}$ of SIN-1 exposure (Fig. $4 \mathrm{~B}$ ). To further test whether ATP depletion induced by SIN-1 was mediated by the release of zinc from intracellular stores and responsible for the neurotoxicity, we examined the effect of TPEN on ATP depletion and neurotoxicity induced by a short period of SIN-1 exposure. TPEN at $1 \mu \mathrm{M}$ had no effect on ATP depletion induced by SIN-1 (1 mM for $1 \mathrm{hr}$ ) (Fig. 4D). TPEN at 3 or $10 \mu \mathrm{M}$ also failed to block ATP depletion (data not shown). However, TPEN $(10 \mu \mathrm{M})$ completely blocked neurotoxicity at $24 \mathrm{hr}$ after $1 \mathrm{hr}$ of SIN-1 exposure (data not shown). These results suggested that ATP depletion induced by peroxynitrite might be irrelevant to the neurotoxicity of peroxynitrite and intracellular zinc release.

\section{TPEN did not block SIN-1-induced GSH depletion}

It has been reported that zinc causes GSH depletion in mixed cortical cultures containing neurons and astrocytes, which later leads to neuronal death (Chen and Liao, 2003). We next tested whether peroxynitrite causes GSH depletion and, if so, whether this depletion could be blocked by TPEN. SIN-1 (1 mM) exposure to neurons for 30 min caused a significant drop in GSH $(p<$ 0.001 ) that was not blocked by TPEN at $10 \mu \mathrm{M}$ (Fig. 5). In four experiments, the cellular levels of GSH in SIN-1 and TPEN plus SIN-1 groups were $49 \pm 7$ and $47 \pm 9 \%$ of control $(p<0.001)$, respectively. This result suggested that GSH depletion was not induced by zinc. Zinc release and GSH depletion may both contribute to the neurotoxicity induced by peroxynitrite.

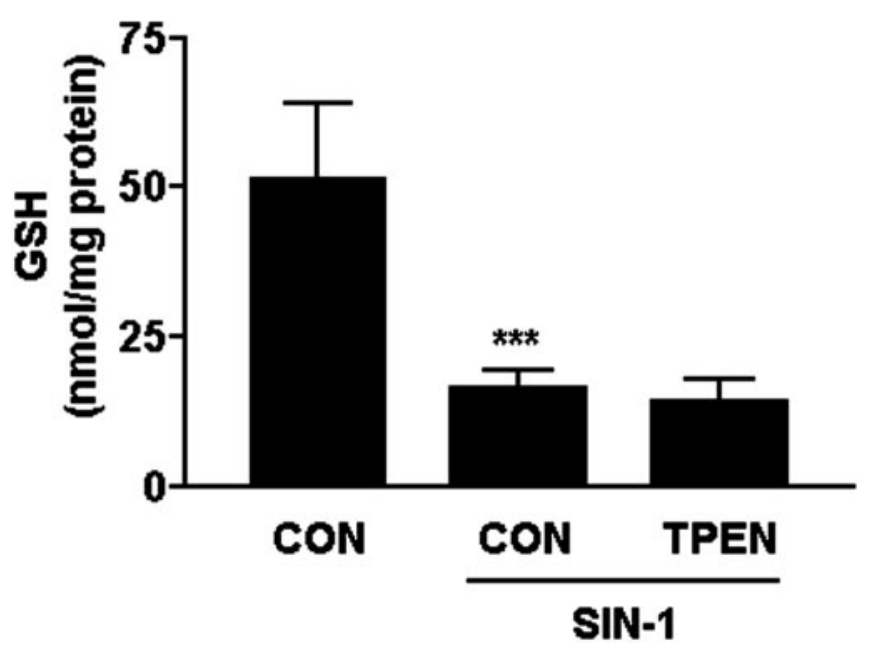

Figure 5. TPEN had no effect on GSH depletion induced by SIN-1. Neurons were exposed to SIN-1 (1 mM) in the absence or presence of TPEN $(10 \mu \mathrm{m})$ for $30 \mathrm{~min}$, and then the neurons were lysed and assayed for GSH activity. One representative experiment of four performed is shown. ${ }^{* * *} p<0.001$ was obtained when the SIN-1 group was compared with the control group. CON, Control. Error bars represent SD.

\section{A}

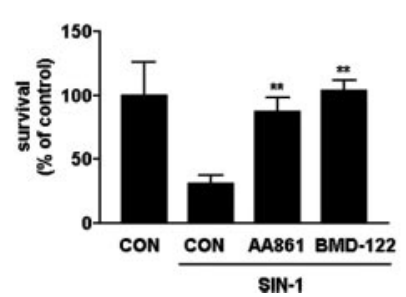

Figure 6. SIN-1-induced neurotoxicity was mediated by 12-LOX activation. $A, 12-\mathrm{LOX}$ inhibitors blocked neurotoxicity induced by SIN-1. Neurons were exposed to SIN-1 (1 mM) for $1 \mathrm{hr}$, and the toxicity was examined at $24 \mathrm{hr}$. For AA861 (10 $\mu \mathrm{M})$ - and BMD-122 (1 $\mu \mathrm{M})$-treated groups, the drugs were applied before, during, and after SIN-1 treatment. ${ }^{* *} p<0.01$ was obtained when drug-treated groups were compared with the group treated with SIN-1 alone. One representative experiment of five performed is shown. $B$, TPEN blocked 12-LOX activation induced by SIN-1. Neurons were exposed to SIN-1 (1 mM) in the absence or presence of TPEN $(10 \mu \mathrm{m})$ for $60 \mathrm{~min}$, and then the 12-LOX activity was examined. ${ }^{* *} p<0.001$ was obtained when the SIN-1-treated group was compared with the control group. ${ }^{\# \#} p<0.01$ was obtained when the TPEN-treated group was compared with the SIN-1 alone group. One representative experiment of four performed is shown. CON, Control. Error bars represent SD.

\section{2-LOX activation is involved in}

peroxynitrite-induced neurotoxicity

Glutathione depletion has been shown to cause 12-LOX activation (Li et al., 1997). It has been suggested that zinc can also participate in the activation of 12-LOX leading to free-radical generation and neuronal injury (Aizenman et al., 2000; Du et al., 2002). We found that two 12-LOX inhibitors, AA861 at $10 \mu \mathrm{M}(\mathrm{Li}$ et al., 1997) and BMD-122 at $1 \mu \mathrm{M}$ (Honn et al., 1994; Nie et al., 2000), did not block neurotoxicity induced by $24 \mathrm{hr}$ of SIN-1 exposure (data not shown). However, they significantly blocked the neurotoxicity at $24 \mathrm{hr}$ after a short period ( $1 \mathrm{hr}$ ) of SIN-1 exposure (Fig. 6A). In the presence of AA861 and BMD-122, neuronal survival was $73 \pm 7$ and $94 \pm 5 \%$, respectively, compared with neuronal survival in the presence of SIN-1 alone ( $37 \pm$ $4 \% ; p<0.01 ; n=5)$. To rule out the possibility that the protective effects of AA861 and BMD-122 might be attributable to their chelation of zinc, equimolar concentrations of zinc together with AA861 or BMD-122 were also tested and found to still protect 


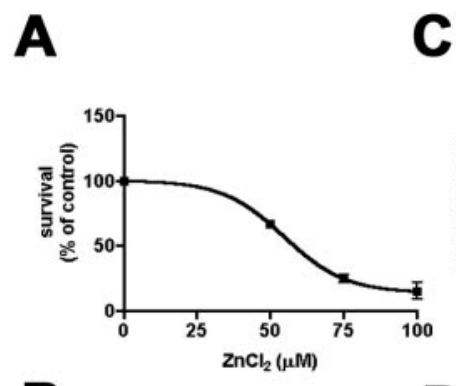

C

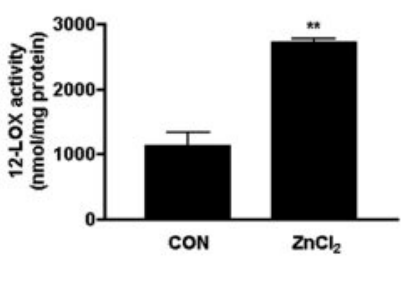

B

D
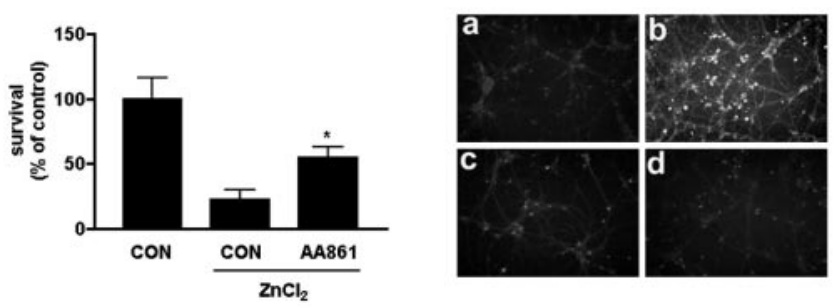

Figure 7. 12-LOX activation is involved in neurotoxicity induced by exogenous zinc. $A$, Concentration dependence of zinc toxicity to neurons. Neurons were exposed to a various concentration of $\mathrm{ZnCl}_{2}(50,75$, and $100 \mu \mathrm{m})$ for $90 \mathrm{~min}$, and the neurotoxicity was examined at $24 \mathrm{hr}$. One representative experiment of five performed is shown. $B, 12-\mathrm{LOX}$ inhibitor blocked neurotoxicity induced by zinc. Neurons were exposed to $\mathrm{ZnCl}_{2}(75 \mu \mathrm{m})$ for $90 \mathrm{~min}$, and the toxicity was examined at $24 \mathrm{hr}$. For the drug-treated group, AA861 (10 $\mu \mathrm{M})$ was applied before, during, and after zinc treatment. ${ }^{*} p<0.05$ was obtained when the drug-treated group was compared with the group treated with zinc alone. One representative experiment of three performed is shown. C, Zinc induced 12-LOX activation. Neurons were exposed to $\mathrm{ZnCl}_{2}(75 \mu \mathrm{m})$ for $1 \mathrm{hr}$, and then the 12 -LOX activity was examined. ${ }^{* *} p<0.01$ was obtained when the zinc-treated group was compared with the control group. One representative experiment of three performed is shown. $D$, Inhibition of 12-LOX blocked ROS generation induced by zinc. At $2 \mathrm{hr}$ after zinc exposure (75 $\mu \mathrm{M}$ for $90 \mathrm{~min}$ ), neurons were loaded with DHR-123 for $20 \mathrm{~min}$, and the image was examined under a fluorescent microscope. $a$, Control; $b, \mathrm{ZnCl}_{2} ; c, \mathrm{AA} 861 / \mathrm{ZnCl}_{2}$; $d$, vitamin $\mathrm{E} / \mathrm{ZnCl}_{2}$. One representative experiment of three performed is shown. CON, Control. Error bars represent SD.

against SIN-1-induced toxicity (data not shown). This result suggested that the metabolism of arachidonic acid via 12-LOX is involved in peroxynitrite-induced neurotoxicity. To examine the specificity of 12-LOX activation, we also examined the effect of two 5-LOX inhibitors, MK-886 (1 $\mu \mathrm{M})$ and caffeic acid $(30 \mu \mathrm{M})$, on the neurotoxicity induced by SIN-1 and found that both drugs had no protective effect (data not shown). The inhibitor of cyclooxygenase (COX), indomethacin, and the inhibitors of cytochrome $\mathrm{P} 450$, proadifen and clotrimazole, also had no protective effect (data not shown), suggesting that the metabolic pathways of arachidonic acid via COX and cytochrome P450 (Shimizu and Wolfe, 1990) were not involved in the neurotoxicity induced by peroxynitrite. To further examine the involvement of 12-LOX in peroxynitrite-induced neurotoxicity, we assayed the cellular levels of 12-LOX activity after 60 min of SIN-1 (1 mM) exposure to neurons. SIN-1 caused significant activation of 12-LOX (Fig. $6 B)$. TPEN at $10 \mu \mathrm{M}$ blocked 12-LOX activation (Fig. $6 B$ ). In four experiments, the levels of 12-LOX activity were $170 \pm$ $16 \%$ of the control levels $(p<0.01)$.

\section{Zinc-induced neurotoxicity was mediated by activation of 12-LOX}

The finding that TPEN blocked SIN-1-induced 12-LOX activation suggests that the liberation of intracellular zinc by peroxynitrite is upstream of 12-LOX. Therefore, inhibition of 12-LOX activity might be able to block the toxicity of exogenously applied
A

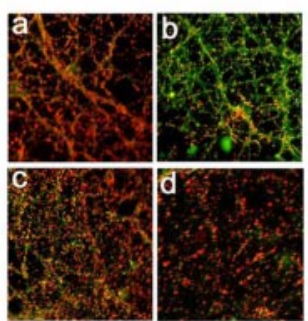

B

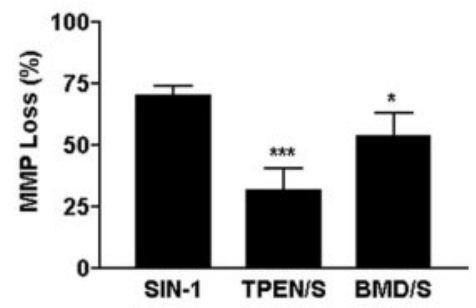

Figure 8. The zinc chelator and a 12-LOX inhibitor attenuated the mitochondrial membrane depolarization induced by peroxynitrite. TPEN (10 $\mu \mathrm{m})$ and BMD-122 $(1 \mu \mathrm{m})$ attenuated mitochondrial membrane depolarization induced by SIN-1. A, At $5 \mathrm{hr}$ after SIN-1 exposure ( $1 \mathrm{~mm}$ for $90 \mathrm{~min}$ ), neurons were loaded with JC-1 for $20 \mathrm{~min}$, and the image was examined under a fluorescent microscope. $a$, Control; $b$, SIN-1; $c$, TPEN/SIN-1; $d$, BMD-122/SIN-1. The percentage decrease of $\mathrm{JC}-1$ ratio reflecting the loss of mitochondrial membrane potential is shown in $B$. ${ }^{*} p<0.05$ and $^{* * *} p<0.001$ were obtained when BMD-122- or TPEN-treated neurons were compared with neurons treated with SIN-1 alone. One representative experiment of three performed is shown. Error bars represent SD. TPEN/S, TPEN/SIN-1; BMD/S, BMD-122/SIN-1.

zinc. Indeed, zinc caused concentration-dependent neurotoxicity at $24 \mathrm{hr}$ after neurons were exposed to $\mathrm{ZnCl}_{2}$ for $90 \mathrm{~min}$ (Fig. $7 A)$. The $\mathrm{EC}_{50}$ value of zinc-induced neurotoxicity was $58 \pm 5 \mu \mathrm{M}$ $(n=5)$. The toxicity induced by $\mathrm{ZnCl}_{2}(75 \mu \mathrm{M})$ was significantly attenuated by AA861 (10 $\mu \mathrm{M})$ (Fig. $7 B)$. When $\mathrm{ZnCl}_{2}$ was applied to neurons, a significant increase in 12-LOX activity was observed (Fig. 7C). In three experiments, the 12-LOX activity was $197 \pm$ $14 \%$ of control levels $(p<0.01)$.

Oxidation of arachidonic acid by 12-LOX can generate lipid peroxides (Chamulitrat et al., 1991; Dikalov and Mason, 2001) and cause neurotoxicity (Li et al., 1997). At $2 \mathrm{hr}$ after $90 \mathrm{~min}$ of $\mathrm{ZnCl}_{2}$ exposure, increased ROS generation was observed in neurons (Fig. 7D), indicated by enhanced DHR-123 fluorescence. AA861 significantly attenuated ROS generation after zinc exposure (Fig. 7D). BMD-122 also had a similar effect (data not shown). As a positive control, we found that the commonly used antioxidant vitamin $\mathrm{E}(10 \mu \mathrm{M})$ also blocked ROS generation induced by zinc (Fig. 7D).

Peroxynitrite-induced mitochondrial membrane depolarization was partially blocked by zinc chelation and 12-LOX inhibition

It has been reported that both peroxynitrite and zinc can cause mitochondrial membrane depolarization, permeability transition pore opening, and the release of factors capable of initiating apoptosis (Jiang et al., 2001; Sensi et al., 2003; Szabo, 2003). Whether the mitochondrial membrane depolarization induced by peroxynitrite is mediated by intracellular zinc release is unknown. We found that SIN-1 induced a significant loss of $\Psi \mathrm{m}$, indicated by enhanced green fluorescence of JC-1 and diminished red fluorescence formed by JC-1 aggregates in mitochondria of neurons compared with control neurons (Fig. 8). TPEN and BMD-122 partially attenuated the loss of $\Psi \mathrm{m}$ (Fig. 8). In three experiments, the loss of $\Psi \mathrm{m}$ in the presence of SIN-1 was $67 \pm 4 \%$; when TPEN and BMD-122 were coapplied, the loss of $\Psi \mathrm{m}$ was $27 \pm 3 \%(p<0.001)$ and $48 \pm 3 \%(p<0.05)$, respectively.

\section{Peroxynitrite- or zinc-induced neuronal apoptosis}

The duration of SIN-1 exposure is critical for the mode of cell death (Bonfoco et al., 1995; Zhang and Rosenberg, 2004). We 


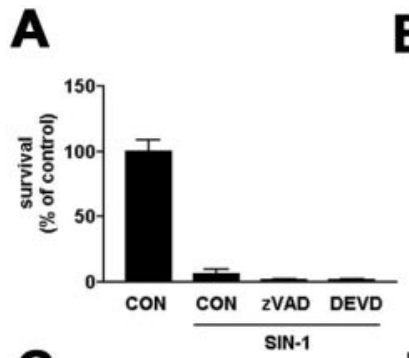

C

B
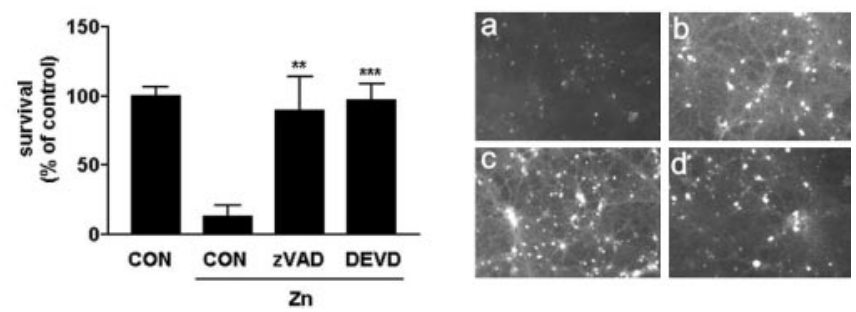

Figure 9. Peroxynitrite or zinc induced caspase-3 activation. A, Caspase inhibitors did not block neurotoxicity induced by $24 \mathrm{hr}$ of SIN-1 exposure. Neurons were exposed to SIN- 1 for $24 \mathrm{hr}$, and then toxicity was examined. Ac-DEVD-CMK and zVAD-FMK were administered before and during SIN-1 exposure. B, Caspase inhibitors blocked neurotoxicity induced by short exposure of SIN-1. Neurons were exposed to SIN-1 for $90 \mathrm{~min}$, and toxicity was examined at $24 \mathrm{hr}$. Ac-DEVDCMK and zVAD-FMK were administered before, during, and after SIN- 1 exposure. ${ }^{*} p<0.05$ and ${ }^{* * *} p<0.001$ were obtained when drug-treated groups were compared with the group treated with SIN-1 alone. One representative experiment of four performed is shown. C, Caspase inhibitors blocked neurotoxicity induced by zinc. Neurons were exposed to $\mathrm{ZnCl}_{2}$ for 90 min, and toxicity was examined at $24 \mathrm{hr}$. Ac-DEVD-CMK and ZVAD-FMK were administered before, during, and after $\mathrm{ZnCl}_{2}$ exposure. ${ }^{* *} p<0.01$ and ${ }^{* * *} p<0.001$ were obtained when drug-treated groups were compared with the group treated with $\mathrm{ZnCl}_{2}$ alone. One representative experiment of four performed is shown. D, SIN-1 or $\mathrm{ZnCl}_{2}$ induced caspase-3 activation. At $7 \mathrm{hr}$ after SIN-1 exposure (1 mm for $90 \mathrm{~min}$ ), neurons were loaded with FITC-DEVD-FMK for $1 \mathrm{hr}$, and then the image was examined under fluorescent microcopy. $a$, Control; $b, \operatorname{SIN}-1 ; c, \mathrm{ZnCl}_{2} ; d$, staurosporine. One representative experiment of three performed is shown. CON, Control. Error bars represent SD.

examined whether caspase activation is involved in the neurotoxicity induced by a long period $(24 \mathrm{hr})$ as well as a short period ( 1 hr) of exposure to SIN-1. A caspase-3 inhibitor, Ac-DEVD-CMK at $100 \mu \mathrm{M}$, had no effect on neurotoxicity induced by $24 \mathrm{hr}$ of SIN-1 exposure (Fig. 9A) but significantly blocked neurotoxicity at $24 \mathrm{hr}$ after $1 \mathrm{hr}$ of SIN-1 exposure (Fig. 9B). Neuronal survival in the presence of Ac-DEVD-CMK was $78 \pm 6 \%(n=4)$ of control, which was significantly higher $(p<0.001)$ than neuronal survival with SIN-1 treatment alone $(29 \pm 6 \%)$. A pancaspase inhibitor, zVAD-FMK $(100 \mu \mathrm{M})$, also failed to block the neurotoxicity induced by SIN-1 after $24 \mathrm{hr}$ of exposure (Fig. 9A). However, when neurons were exposed to SIN-1 for $1 \mathrm{hr}$, the toxicity at $24 \mathrm{hr}$ was blocked by zVAD-FMK (Fig. 9B). Neuronal survival in the presence of $z \mathrm{VAD}-\mathrm{FMK}$ was $52 \pm 7 \%$, significantly higher than in the SIN-1-treated group $(p<0.05 ; n=4)$. AcDEVD-CMK and zVAD-FMK also protected against neurotoxicity at $24 \mathrm{hr}$ after $90 \mathrm{~min}$ of zinc $(75 \mu \mathrm{M})$ exposure (Fig. 9C). Consistent with these pharmacological data, caspase activation was observed at $7 \mathrm{hr}$ after $90 \mathrm{~min}$ of exposure of neurons to SIN-1 or zinc (Fig. 9D). As a positive control, the activation of caspase induced by staurosporine ( $1 \mu \mathrm{M}$ for $5 \mathrm{hr}$ ), an apoptosis-inducing agent, was also examined (Fig. 9D). These results suggested that peroxynitrite causes caspase-dependent apoptosis, which is likely mediated by zinc release from intracellular stores.
A
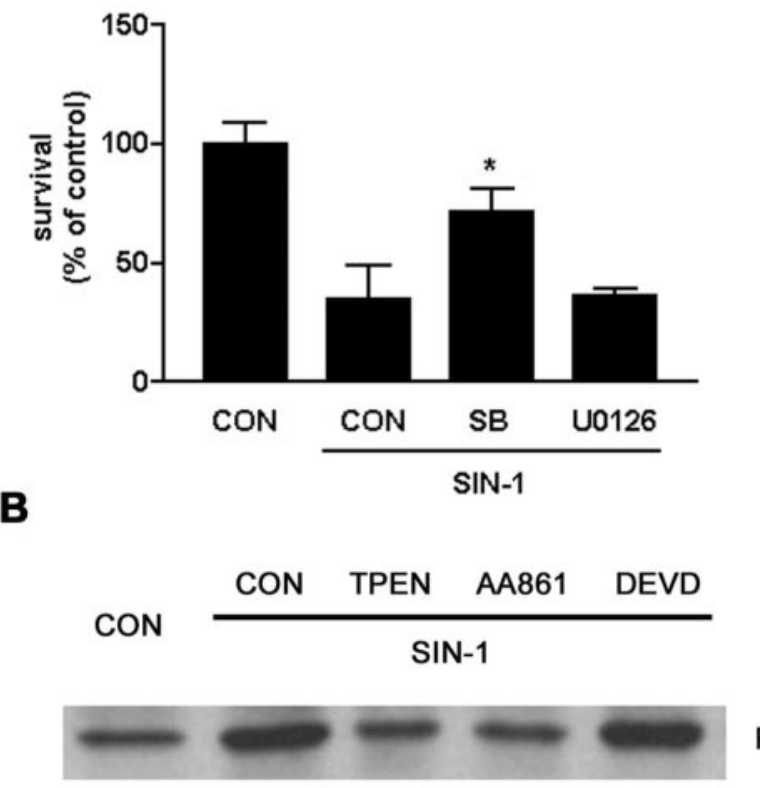

P-p38

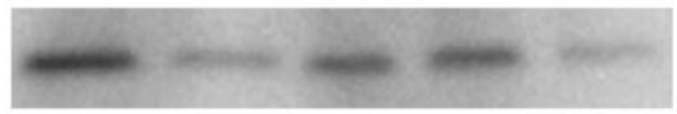

p38

C

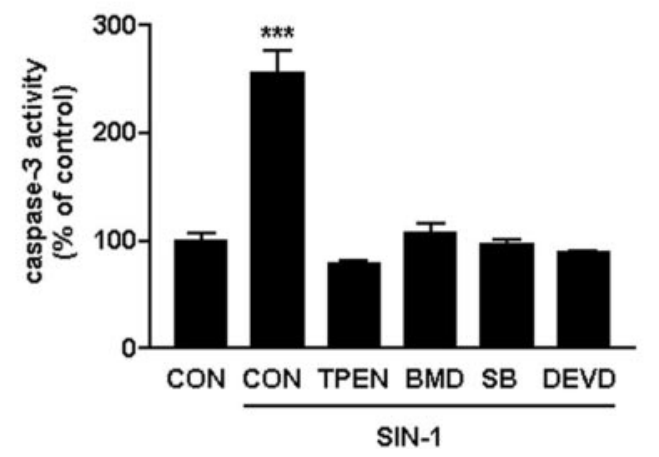

Figure 10. Peroxynitrite induced phosphorylation of $\mathrm{p} 38$ MAPK. A, Inhibition of $\mathrm{p} 38$, but not ERK42/44, blocked neurotoxicity induced by SIN-1. Neurons were exposed to SIN- 1 for $1 \mathrm{hr}$, and toxicity was examined at $24 \mathrm{hr}$. SB203580 (SB) $(10 \mu \mathrm{M})$ and U0126 (5 $\mu \mathrm{M})$ were administered before, during, and after SIN-1 exposure. ${ }^{*} p<0.05$ was obtained when the SB-treated group was compared with the SIN-1 group. One representative experiment of four performed is shown. $B, S I N-1$ induced phosphorylation of $p 38$. Neurons were exposed to SIN-1 in the absence or presence of TPEN, AA861, and AC-DEVD-CMK for $1 \mathrm{hr}$, and the phosphorylation of p38 was examined by immunoblot. SIN-1 (1 mm) induced significant phosphorylation of p38 (P-p38). TPEN (10 $\mu \mathrm{m}$ ) and AA861 (10 $\mu \mathrm{m})$ blocked the phosphorylation, but Ac-DEVD-CMK (DEVD) (100 $\mu \mathrm{M}$ ) had no effect. One representative experiment of three performed is shown. $C$, Inhibition of zinc release, 12-LOX activation, and p38 phosphorylation blocked SIN-1-induced caspase activation. At $7 \mathrm{hr}$ after SIN-1 exposure (1 mm for $90 \mathrm{~min}$ ), neurons were lysed. After centrifugation, the caspase- 3 activity in the supernatant was examined. ${ }^{* * *} p<0.001$ was obtained when the SIN-1-treated group was compared with the control or the drug-treated groups. One representative experiment of three performed is shown. CON, Control. Error bars represent SD.

Phosphorylation of p38 mitogen-activated protein kinase is downstream of zinc release and 12-LOX activation and upstream of caspase activation

Activation of mitogen-activated protein kinases (MAPKs) has been found to link oxidative stress and neurotoxicity (McLaughlin et al., 2001; Du et al., 2002; Choi et al., 2004). However, spe- 
cific MAPKs, for example, extracellular signal-regulated kinase (ERK) $42 / 44$ or p38, might be involved in neuronal necrosis or apoptosis induced by different oxidizing agents (McLaughlin et al., 2001; Du et al., 2002). It has been shown previously that an oxidizing agent, DTDP, causes neuronal apoptosis via a $\mathrm{Zn}^{2+}$ p38-K ${ }^{+}$efflux pathway (McLaughlin et al., 2001), whereas methylisothiazolinone, another oxidizing agent, causes nonapoptotic neuronal death via a $\mathrm{Zn}^{2+}$-12-LOX-ROS pathway (Du et al., 2002). We tested whether inhibition of ERK42/44 or p38 activation could block the neurotoxicity induced by SIN-1. 4-(4-Fluorophenyl)-2(4-methylsulfinylphenyl)-5-(4-pyridyl)imidazole (SB203580) (10 $\mu \mathrm{M}$ ), an inhibitor of 38 MAPK (Saklatvala et al., 1996), significantly blocked the neurotoxicity induced by SIN-1 (Fig. 10A). Neuronal survival in the presence of SB203580 was $79 \pm 4 \%$ of control $(n=3)$, significantly higher $(p<0.01)$ than the survival of neurons treated with SIN-1 alone (32 $\pm 4 \%)$. Neuronal survival in the presence of 1,4-diamino-2,3-dicyano-1,4-bis(o-aminophenylmercapto) butadiene (U0126), an inhibitor of ERK42/44 phosphorylation (Du et al., 2002), plus SIN-1 was $36 \pm 2 \%$, which is not significantly different from SIN-1 alone.

At $1 \mathrm{hr}$ after SIN-1 exposure to neurons, significant elevation of p38 phosphorylation was observed (Fig. 10 B). The activation of p38 was also observed when zinc or authentic peroxynitrite was used (data not shown). TPEN and AA861 completely blocked SIN-1-induced phosphorylation of p38, but the caspase-3 inhibitor Ac-DEVD-CMK had no effect (Fig. 10B). This result suggests that caspase activation is downstream of p38 activation. To further test this idea, we measured cellular caspase- 3 activity $7 \mathrm{hr}$ after neurons were exposed to SIN-1 (90 min exposure) in the absence or presence of TPEN, AA861, and SB203580 (Fig. 10C). The activity of caspase-3 was significantly elevated by SIN-1 and attenuated by the coapplication of TPEN, AA861, and SB203580 with SIN-1. As a positive control, AcDEVD-CMK completely blocked SIN-1-induced activation of caspase-3 (Fig. 10C). These results suggested that activation of p38 is downstream of zinc release and 12-LOX activation induced by peroxynitrite.

\section{Discussion}

Generation of peroxynitrite and disruption of zinc homeostasis have recently been considered to be the two major components of neuronal injury in stroke and neurodegenerative diseases, including Alzheimer's disease and ALS (Heales et al., 1999; Weiss et al., 2000). The link between these two pathways of neurodegeneration has not been identified previously. In this study, we found that peroxynitrite-induced neuronal apoptosis is mediated by zinc release from intracellular stores and subsequent activation of 12-lipoxygenase, ROS generation, mitochondrial dysfunction, activation of $\mathrm{p} 38$ MAPK, and caspases.

Peroxynitrite, a reaction product between nitric oxide and superoxide, is the most potent oxidant identified (Beckman et al., 1990; Beckman and Koppenol, 1996). Recently, two oxidizing agents, DTDP and methylisothiazolinone, have been shown to induce the liberation of zinc from intracellular stores, leading to neurotoxicity (Aizenman et al., 2000; Du et al., 2002). In the present study, we found that TPEN, a zinc chelator, protected against neurotoxicity induced by exogenously as well as endogenously generated peroxynitrite. Peroxynitrite-induced liberation of zinc from intracellular stores was identified by using a $\mathrm{Zn}^{2+}$-specific dye, FluoZin-3, or intramolecular FRET. While this manuscript was in revision, Bossy-Wetzel et al. (2004) reported that nitric oxide- or NMDA-induced liberation of zinc from mixed neuronal cultures was mediated by the intracellular generation of peroxynitrite. These results suggest that peroxynitrite causes zinc-mediated neuronal injury.

Impairment of energy metabolism and free-radical generation are two major mechanisms of zinc-induced neurotoxicity (Weiss et al., 2000; Dineley et al., 2003). TPEN did not block ATP depletion induced by peroxynitrite, suggesting that ATP depletion is not mediated by zinc liberated from intracellular stores. The discrepancy between TPEN neuroprotection and its failure to block ATP depletion suggests that the neurotoxicity mediated by intracellular zinc is independent of the early ATP depletion. However, we cannot rule out the possibility that zinc may cause ATP depletion at a later stage and contribute to neuronal injury. Pyruvate, an end product of glycolysis, blocked ATP depletion induced by 1-2 hr of SIN-1 exposure, but not by 3 hr of SIN-1 exposure, suggesting that peroxynitrite might initially inhibit glycolysis and that zinc might contribute to the late mitochondrial dysfunction and the subsequent disturbance of energy production.

Many studies have indicated that ROS play an important role in the toxicity of zinc (Kim et al., 1999; Noh et al., 1999; Sensi et al., 1999), but the mechanism underlying the ROS generation was unclear. Recently, the AA metabolic pathway mediated by 12 LOX activation was found to be an important source of ROS when neurons were exposed to oxidative stress (Li et al., 1997; Aizenman et al., 2000; Stanciu et al., 2000; Khanna et al., 2003). Zinc has been reported to bind to and activate phospholipase $\mathrm{A}_{2}$ $\left(\mathrm{PLA}_{2}\right)$, leading to the release of AA from phospholipids in membrane (Lindahl and Tagesson, 1996). Although AA can be metabolized by COX, LOX, and cytochrome P450 epoxygenase (Shimizu and Wolfe, 1990), the involvement of COX and epoxygenase in the neurotoxicity induced by peroxynitrite was excluded here because inhibitors of these enzymes did not have any protective effect. 12-Lipoxygenase is the primary LOX expressed in the brain (Shimizu and Wolfe, 1990). Our results suggest that the neurotoxicity induced by a short exposure to peroxynitrite is mediated by 12 -LOX but not by 5-LOX. It is interesting to note that although SIN-1 and zinc can both cause activation of 12LOX, the inhibitors of 12 -LOX are more potent in blocking peroxynitrite-induced neurotoxicity than in preventing the toxicity induced by exogenous zinc. The reason for this discrepancy is unclear. It is possible that glutathione depletion and zinc release from intracellular stores after peroxynitrite administration may have an additive or a synergistic effect on 12-LOX activation and thus induce cell death mainly through the 12-LOX pathway. The toxicity induced by exogenous zinc may occur via 12-LOXdependent and -independent pathways.

Recently, it has been reported that nitric oxide and glutathione depletion have a synergistic effect in producing toxicity in primary midbrain cultures (Canals et al., 2003). It is possible, as suggested by Canals et al. (2003), that nitric oxide stimulates the release of AA and therefore provides more substrate for 12-LOX, which is activated by the glutathione depletion. However, in their model system, nitric oxide was added to glutathione-depleted cultures, which might be expected to generate increased amounts of superoxide (Mytilineou et al., 2002). Therefore, the synergistic effect between nitric oxide and glutathione depletion in causing 12-LOX activation and neurotoxicity may be attributable to the formation of peroxynitrite by the reaction of nitric oxide and superoxide.

Interestingly, in oligodendrocyte cultures, nitric oxide was shown to be protective against the toxicity of GSH depletion (Rosenberg et al., 1999). The toxicity in oligodendrocytes has been found to be mediated by an increase in 12-LOX activity (Wang et al., 2004). Nitric oxide has been shown to inhibit 12- 
LOX activity in human and rabbit platelets (Nakatsuka and Osawa, 1994; Fujimoto et al., 1998).

It is not currently known how peroxynitrite or zinc induces 12-LOX activation. We found that two $\mathrm{PLA}_{2}$ inhibitors, quinacrine and DEDA, only had a minor effect in blocking the toxicity of peroxynitrite or zinc (data not shown), suggesting that other signaling molecules may be involved in the activation of 12-LOX. It has been reported that inhibition of protein kinase $C$ (PKC) completely blocks zinc-induced free-radical generation and neurotoxicity (Noh et al., 1999). Activation of PKC was observed at 15 min after zinc exposure (Noh et al., 1999), earlier than the activation of 12-LOX induced by zinc $(60 \mathrm{~min})$ in our current study. Therefore, it is possible that the activation of PKC may be upstream of 12-LOX activation induced by zinc.

Free-radical generation and mitochondrial dysfunction are thought to be the critical effectors of zinc-mediated neurotoxicity (Weiss et al., 2000; Sensi et al., 2003). Zinc released from presynaptic terminals or from intracellular zinc binding proteins may impair the mitochondrial membrane potential and cause freeradical generation and apoptotic neuronal injury (Jiang et al., 2001; Sensi et al., 2003). We found that 12-LOX inhibition completely blocked ROS generation induced by zinc and partially blocked the loss of mitochondrial membrane potential induced by peroxynitrite. These results suggested that ROS might primarily be derived from the activation of 12-LOX and that dysfunction of mitochondria might further facilitate the formation of free radicals (Sensi et al., 1999; Sensi et al., 2003).

Unlike the toxicity induced by $24 \mathrm{hr}$ of SIN-1 exposure, which is caspase independent, a short exposure to SIN-1 (1-2 hr) caused caspase-dependent toxicity (Fig. 9). This result is consistent with a previous report that the mode of cell death induced by peroxynitrite in mixed neuronal cultures is dependent on the severity and duration of peroxynitrite exposure (Bonfoco et al., 1995). We also observed that the toxicity induced by a short zinc exposure is caspase dependent. Zinc release, p38 MAPK activation, and potassium efflux are thought to be upstream of caspase activation in DTDP-induced neuronal apoptosis (McLaughlin et al., 2001). Zinc release, p38 MAPK activation, and potassium efflux were also involved in peroxynitrite-induced neuronal apoptosis (Bossy-Wetzel et al., 2004), but tetraethylammonium, a potassium channel blocker, was ineffective in blocking neurotoxicity (data not shown), suggesting that potassium efflux is not essential for peroxynitrite-induced neuronal apoptosis. Inhibition of ERK42/44 also failed to block peroxynitrite-induced neurotoxicity. Surprisingly, we found that, in contrast to the effect of TPEN, which blocked caspase-dependent apoptosis, as well as the caspase-independent neurotoxicity caused by $24 \mathrm{hr}$ of exposure to SIN-1 (Fig. 1A), the inhibitors of 12-LOX only blocked neuronal apoptosis after a short exposure to SIN-1 (Fig. 6A) (data not shown). These results suggested that 12-LOX activation is crucial for zinc-induced neuronal apoptosis. In caspase-independent neuronal death, the toxicity induced by peroxynitrite appears to be independent of the activation of 12-LOX.

During hypoxic-ischemic brain injury, there is a 10-fold increase in free arachidonic acid, which is generated from phospholipids by the activation of $\mathrm{PLA}_{2}$ (Lipton, 1999). PLA 2 knock-out mice are resistant to hypoxia-ischemia-induced brain injury (Bonventre et al., 1997), suggesting that AA metabolism might be critically involved in the brain injury. Inhibitors of LOX have been shown to have protective effects against oxygen-glucose deprivation-induced neurotoxicity to rat hippocampal slice cultures (Arai et al., 2001), as well as ischemia-induced neuronal death in vivo (Nakagomi et al., 1989), suggesting that the activa- tion of 12-LOX might be an important cause of ischemic brain injury.

It has been shown recently that overexpression of the zinc binding proteins metallothionein I, II, and III can extend the survival of ALS transgenic mouse (Puttaparthi et al., 2002), suggesting that intracellular free zinc is toxic to motor neurons. This observation is also in harmony with the theory that peroxynitrite toxicity is one of the major pathogenic mechanisms of ALS (Beckman et al., 2001). It will be interesting to determine whether blockade of 12-LOX activity might also have a therapeutic effect in improving the survival of ALS transgenic mice. Our results suggest that zinc homeostasis and the 12-LOX metabolic pathway may play an important role in the pathogenesis of a variety of acute and chronic neurodegenerative diseases in which peroxynitrite is involved.

\section{References}

Aizenman E, Stout AK, Hartnett KA, Dineley KE, McLaughlin B, Reynolds IJ (2000) Induction of neuronal apoptosis by thiol oxidation: putative role of intracellular zinc release. J Neurochem 75:1878-1888.

Ara J, Przedborski S, Naini AB, Jackson-Lewis V, Trifiletti RR, Horwitz J, Ischiropoulos H (1998) Inactivation of tyrosine hydroxylase by nitration following exposure to peroxynitrite and 1-methyl-4-phenyl-1,2,3,6tetrahydropyridine (MPTP). Proc Natl Acad Sci USA 95:7659-7663.

Arai K, Nishiyama N, Matsuki N, Ikegaya Y (2001) Neuroprotective effects of lipoxygenase inhibitors against ischemic injury in rat hippocampal slice cultures. Brain Res 904:167-172.

Aravindakumar CT, Ceulemans J, De Ley M (1999) Nitric oxide induces Zn2+ release from metallothionein by destroying zinc-sulphur clusters without concomitant formation of S-nitrosothiol. Biochem J 344:253-258.

Armstrong C, Leong W, Lees GJ (2001) Comparative effects of metal chelating agents on the neuronal cytotoxicity induced by copper $(\mathrm{Cu}+2)$, iron $(\mathrm{Fe}+3)$ and zinc in the hippocampus. Brain Res 892:51-62.

Back SA, Gan X, Li Y, Rosenberg PA, Volpe JJ (1998) Maturationdependent vulnerability of oligodendrocytes to oxidative stress-induced death caused by glutathione depletion. J Neurosci 18:6241-6253.

Back SA, Khan R, Gan X, Rosenberg PA, Volpe JJ (1999) A new Alamar Blue viability assay to rapidly quantify oligodendrocyte death. J Neurosci Methods 91:47-54.

Beckman JS, Koppenol WH (1996) Nitric oxide, superoxide, and peroxynitrite: the good, the bad, and ugly. Am J Physiol 271:C1424-C1437.

Beckman JS, Beckman TW, Chen J, Marshall PA, Freeman BA (1990) Apparent hydroxyl radical production by peroxynitrite: implications for endothelial injury from nitric oxide and superoxide. Proc Natl Acad Sci USA 87:1620-1624.

Beckman JS, Estevez AG, Crow JP, Barbeito L (2001) Superoxide dismutase and the death of motoneurons in ALS. Trends Neurosci 24:S15-S20.

Berendji D, Kolb-Bachofen V, Meyer KL, Grapenthin O, Weber H, Wahn V, Kroncke KD (1997) Nitric oxide mediates intracytoplasmic and intranuclear zinc release. FEBS Lett 405:37-41.

Bonfoco E, Krainc D, Ankarcrona M, Nicotera P, Lipton SA (1995) Apoptosis and necrosis: two distinct events induced, respectively, by mild and intense insults with $\mathrm{N}$-methyl-D-aspartate or nitric oxide/superoxide in cortical cell cultures. Proc Natl Acad Sci USA 92:7162-7166.

Bonventre JV, Huang Z, Taheri MR, O'Leary E, Li E, Moskowitz MA, Sapirstein A (1997) Reduced fertility and postischaemic brain injury in mice deficient in cytosolic phospholipase A2. Nature 390:622-625.

Bossy-Wetzel E, Talantova MV, Lee WD, Scholzke MN, Harrop A, Mathews E, Gotz T, Han J, Ellisman MH, Perkins GA, Lipton SA (2004) Crosstalk between nitric oxide and zinc pathways to neuronal cell death involving mitochondrial dysfunction and p38-activated $\mathrm{K}+$ channels. Neuron 41:351-365.

Canals S, Casarejos MJ, de Bernardo S, Rodriguez-Martin E, Mena MA (2003) Nitric oxide triggers the toxicity due to glutathione depletion in midbrain cultures through 12-lipoxygenase. J Biol Chem 278:21542-21549.

Canzoniero LM, Turetsky DM, Choi DW (1999) Measurement of intracellular free zinc concentrations accompanying zinc-induced neuronal death. J Neurosci 19:RC31(1-6).

Chabrier PE, Demerle-Pallardy C, Auguet M (1999) Nitric oxide synthases: 
targets for therapeutic strategies in neurological diseases. Cell Mol Life Sci 55:1029-1035.

Chamulitrat W, Hughes MF, Eling TE, Mason RP (1991) Superoxide and peroxyl radical generation from the reduction of polyunsaturated fatty acid hydroperoxides by soybean lipoxygenase. Arch Biochem Biophys 290:153-159.

Chen CJ, Liao SL (2003) Zinc toxicity on neonatal cortical neurons: involvement of glutathione chelation. J Neurochem 85:443-453.

Choi WS, Eom DS, Han BS, Kim WK, Han BH, Choi EJ, Oh TH, Markelonis GJ, Cho JW, Oh YJ (2004) Phosphorylation of 38 MAPK induced by oxidative stress is linked to activation of both caspase-8- and -9-mediated apoptotic pathways in dopaminergic neurons. J Biol Chem 279:20451-20460.

Crow JP, Ye YZ, Strong M, Kirk M, Barnes S, Beckman JS (1997) Superoxide dismutase catalyzes nitration of tyrosines by peroxynitrite in the rod and head domains of neurofilament-L. J Neurochem 69:1945-1953.

Dichter MA (1978) Rat cortical neurons in cell culture: culture methods, cell morphology, electrophysiology, and synapse formation. Brain Res 149:279-293.

Dikalov SI, Mason RP (2001) Spin trapping of polyunsaturated fatty acidderived peroxyl radicals: reassignment to alkoxyl radical adducts. Free Radic Biol Med 30:187-197.

Dineley KE, Votyakova TV, Reynolds IJ (2003) Zinc inhibition of cellular energy production: implications for mitochondria and neurodegeneration. J Neurochem 85:563-570.

Du S, McLaughlin B, Pal S, Aizenman E (2002) In vitro neurotoxicity of methylisothiazolinone, a commonly used industrial and household biocide, proceeds via a zinc and extracellular signal-regulated kinase mitogen-activated protein kinase-dependent pathway. J Neurosci 22:7408-7416.

Eliasson MJ, Sampei K, Mandir AS, Hurn PD, Traystman RJ, Bao J, Pieper A, Wang ZQ, Dawson TM, Snyder SH, Dawson VL (1997) Poly(ADPribose) polymerase gene disruption renders mice resistant to cerebral ischemia. Nat Med 3:1089-1095.

Frederickson CJ (1989) Neurobiology of zinc and zinc-containing neurons. Int Rev Neurobiol 31:145-238.

Fujimoto Y, Tagano S, Ogawa K, Sakuma S, Fujita T (1998) Comparison of the effects of nitric oxide and peroxynitrite on the 12-lipoxygenase and cyclooxygenase metabolism of arachidonic acid in rabbit platelets. Prostaglandins Leukot Essent Fatty Acids 59:95-100.

Gee KR, Zhou ZL, Qian WJ, Kennedy R (2002) Detection and imaging of zinc secretion from pancreatic beta-cells using a new fluorescent zinc indicator. J Am Chem Soc 124:776-778.

Heales SJ, Bolanos JP, Stewart VC, Brookes PS, Land JM, Clark JB (1999) Nitric oxide, mitochondria and neurological disease. Biochim Biophys Acta 1410:215-228.

Hewett SJ, Csernansky CA, Choi DW (1994) Selective potentiation of NMDA-induced neuronal injury following induction of astrocytic iNOS. Neuron 13:487-494.

Hogg N, Darley-Usmar VM, Wilson MT, Moncada S (1992) Production of hydroxyl radicals from the simultaneous generation of superoxide and nitric oxide. Biochem J 281:419-424.

Honn KV, Tang DG, Grossi IM, Renaud C, Duniec ZM, Johnson CR, Diglio CA (1994) Enhanced endothelial cell retraction mediated by 12(S)HETE: a proposed mechanism for the role of platelets in tumor cell metastasis. Exp Cell Res 210:1-9.

Jiang D, Sullivan PG, Sensi SL, Steward O, Weiss JH (2001) Zn(2+) induces permeability transition pore opening and release of pro-apoptotic peptides from neuronal mitochondria. J Biol Chem 276:47524-47529.

Jornot L, Petersen H, Junod AF (1998) Hydrogen peroxide-induced DNA damage is independent of nuclear calcium but dependent on redox-active ions. Biochem J 335:85-94.

Khanna S, Roy S, Ryu H, Bahadduri P, Swaan PW, Ratan RR, Sen CK (2003) Molecular basis of vitamin $\mathrm{E}$ action. Tocotrienol modulates 12lipoxygenase, a key mediator of glutamate-induced neurodegeneration. J Biol Chem 278:43508-43515.

Kim EY, Koh JY, Kim YH, Sohn S, Joe E, Gwag BJ (1999) Zn2+ entry produces oxidative neuronal necrosis in cortical cell cultures. Eur J Neurosci 11:327-334.

Koh JY, Suh SW, Gwag BJ, He YY, Hsu CY, Choi DW (1996) The role of zinc in selective neuronal death after transient global cerebral ischemia. Science 272:1013-1016.

Kroncke KD, Fehsel K, Schmidt T, Zenke FT, Dasting I, Wesener JR, Better- mann H, Breunig KD, Kolb-Bachofen V (1994) Nitric oxide destroys zinc-sulfur clusters inducing zinc release from metallothionein and inhibition of the zinc finger-type yeast transcription activator LAC9. Biochem Biophys Res Commun 200:1105-1110.

LeBel CP, Ischiropoulos H, Bondy SC (1992) Evaluation of the probe $2^{\prime}, 7^{\prime}$ dichlorofluorescin as an indicator of reactive oxygen species formation and oxidative stress. Chem Res Toxicol 5:227-231.

Leist M, Single B, Castoldi AF, Kuhnle S, Nicotera P (1997) Intracellular adenosine triphosphate (ATP) concentration: a switch in the decision between apoptosis and necrosis. J Exp Med 185:1481-1486.

Li Y, Maher P, Schubert D (1997) A role for 12-lipoxygenase in nerve cell death caused by glutathione depletion. Neuron 19:453-463.

Lindahl M, Tagesson C (1996) Zinc (Zn2+) binds to and stimulates the activity of group I but not group II phospholipase A2. Inflammation 20:599-611.

Lipton P (1999) Ischemic cell death in brain neurons. Physiol Rev 79:1431-1568.

Maret W, Vallee BL (1998) Thiolate ligands in metallothionein confer redox activity on zinc clusters. Proc Natl Acad Sci USA 95:3478-3482.

McCabe Jr MJ, Jiang SA, Orrenius S (1993) Chelation of intracellular zinc triggers apoptosis in mature thymocytes. Lab Invest 69:101-110.

McGahon AJ, Martin SJ, Bissonnette RP, Mahboubi A, Shi Y, Mogil RJ, Nishioka WK, Green DR (1995) The end of the (cell) line: methods for the study of apoptosis in vitro. Methods Cell Biol 46:153-185.

McLaughlin B, Pal S, Tran MP, Parsons AA, Barone FC, Erhardt JA, Aizenman E (2001) p38 activation is required upstream of potassium current enhancement and caspase cleavage in thiol oxidant-induced neuronal apoptosis. J Neurosci 21:3303-3311.

Miyawaki A, Llopis J, Heim R, McCaffery JM, Adams JA, Ikura M, Tsien RY (1997) Fluorescent indicators for Ca2+ based on green fluorescent proteins and calmodulin. Nature 388:882-887.

Mytilineou C, Kramer BC, Yabut JA (2002) Glutathione depletion and oxidative stress. Parkinsonism Relat Disord 8:385-387.

Nakagomi T, Sasaki T, Kirino T, Tamura A, Noguchi M, Saito I, Takakura K (1989) Effect of cyclooxygenase and lipoxygenase inhibitors on delayed neuronal death in the gerbil hippocampus. Stroke 20:925-929.

Nakatsuka M, Osawa Y (1994) Selective inhibition of the 12-lipoxygenase pathway of arachidonic acid metabolism by L-arginine or sodium nitroprusside in intact human platelets. Biochem Biophys Res Commun 200:1630-1634.

Nie D, Tang K, Diglio C, Honn KV (2000) Eicosanoid regulation of angiogenesis: role of endothelial arachidonate 12-lipoxygenase. Blood 95:2304-2311.

Noh KM, Kim YH, Koh JY (1999) Mediation by membrane protein kinase C of zinc-induced oxidative neuronal injury in mouse cortical cultures. J Neurochem 72:1609-1616.

Outten CE, O'Halloran TV (2001) Femtomolar sensitivity of metalloregulatory proteins controlling zinc homeostasis. Science 292:2488-2492.

Pal S, Hartnett KA, Nerbonne JM, Levitan ES, Aizenman E (2003) Mediation of neuronal apoptosis by Kv2.1-encoded potassium channels. J Neurosci 23:4798-4802.

Pal S, He K, Aizenman E (2004) Nitrosative stress and potassium channelmediated neuronal apoptosis: is zinc the link? Pflügers Arch 448:296-303

Patel M, Day BJ, Crapo JD, Fridovich I, McNamara JO (1996) Requirement for superoxide in excitotoxic cell death. Neuron 16:345-355.

Pearce LL, Gandley RE, Han W, Wasserloos K, Stitt M, Kanai AJ, McLaughlin MK, Pitt BR, Levitan ES (2000) Role of metallothionein in nitric oxide signaling as revealed by a green fluorescent fusion protein. Proc Natl Acad Sci USA 97:477-482.

Puttaparthi K, Gitomer WL, Krishnan U, Son M, Rajendran B, Elliott JL (2002) Disease progression in a transgenic model of familial amyotrophic lateral sclerosis is dependent on both neuronal and non-neuronal zinc binding proteins. J Neurosci 22:8790-8796.

Radi R, Beckman JS, Bush KM, Freeman BA (1991) Peroxynitrite-induced membrane lipid peroxidation: the cytotoxic potential of superoxide and nitric oxide. Arch Biochem Biophys 288:481-487.

Rosenberg PA (1991) Accumulation of extracellular glutamate and neuronal death in astrocyte-poor cortical cultures exposed to glutamine. Glia 4:91-100.

Rosenberg PA, Aizenman E (1989) Hundred-fold increase in neuronal vulnerability to glutamate toxicity in astrocyte-poor cultures of rat cerebral cortex. Neurosci Lett 103:162-168. 
Rosenberg PA, Amin S, Leitner M (1992) Glutamate uptake disguises neurotoxic potency of glutamate agonists in cerebral cortex in dissociated cell culture. J Neurosci 12:56-61.

Rosenberg PA, Li Y, Ali S, Altiok N, Back SA, Volpe JJ (1999) Intracellular redox state determines whether nitric oxide is toxic or protective to rat oligodendrocytes in culture. J Neurochem 73:476-484.

Rosenberg PA, Li Y, Le M, Zhang Y (2000) Nitric oxide-stimulated increase in extracellular adenosine accumulation in rat forebrain neurons in culture is associated with ATP hydrolysis and inhibition of adenosine kinase activity. J Neurosci 20:6294-6301.

Royall JA, Ischiropoulos H (1993) Evaluation of $2^{\prime}, 7^{\prime}$-dichlorofluorescin and dihydrorhodamine 123 as fluorescent probes for intracellular $\mathrm{H} 2 \mathrm{O} 2$ in cultured endothelial cells. Arch Biochem Biophys 302:348-355.

Saklatvala J, Rawlinson L, Waller RJ, Sarsfield S, Lee JC, Morton LF, Barnes MJ, Farndale RW (1996) Role for p38 mitogen-activated protein kinase in platelet aggregation caused by collagen or a thromboxane analogue. J Biol Chem 271:6586-6589.

Salvioli S, Ardizzoni A, Franceschi C, Cossarizza A (1997) JC-1, but not DiOC6(3) or rhodamine 123, is a reliable fluorescent probe to assess delta psi changes in intact cells: implications for studies on mitochondrial functionality during apoptosis. FEBS Lett 411:77-82.

Sengpiel B, Preis E, Krieglstein J, Prehn JH (1998) NMDA-induced superoxide production and neurotoxicity in cultured rat hippocampal neurons: role of mitochondria. Eur J Neurosci 10:1903-1910.

Sensi SL, Yin HZ, Carriedo SG, Rao SS, Weiss JH (1999) Preferential Zn2+ influx through $\mathrm{Ca} 2+$-permeable AMPA/kainate channels triggers prolonged mitochondrial superoxide production. Proc Natl Acad Sci USA 96:2414-2419.

Sensi SL, Ton-That D, Sullivan PG, Jonas EA, Gee KR, Kaczmarek LK, Weiss $\mathrm{JH}$ (2003) Modulation of mitochondrial function by endogenous $\mathrm{Zn} 2+$ pools. Proc Natl Acad Sci USA 100:6157-6162.

Sheline CT, Behrens MM, Choi DW (2000) Zinc-induced cortical neuronal death: contribution of energy failure attributable to loss of $\mathrm{NAD}(+)$ and inhibition of glycolysis. J Neurosci 20:3139-3146.

Shimizu T, Wolfe LS (1990) Arachidonic acid cascade and signal transduction. J Neurochem 55:1-15.

Stanciu M, Wang Y, Kentor R, Burke N, Watkins S, Kress G, Reynolds I, Klann E, Angiolieri MR, Johnson JW, DeFranco DB (2000) Persistent activa- tion of ERK contributes to glutamate-induced oxidative toxicity in a neuronal cell line and primary cortical neuron cultures. J Biol Chem 275:12200-12206.

Stefanis L, Park DS, Friedman WJ, Greene LA (1999) Caspase-dependent and -independent death of camptothecin-treated embryonic cortical neurons. J Neurosci 19:6235-6247.

Szabo C (1996) The pathophysiological role of peroxynitrite in shock, inflammation, and ischemia-reperfusion injury. Shock 6:79-88.

Szabo C (2003) Multiple pathways of peroxynitrite cytotoxicity. Toxicol Lett 140-141:105-112.

Szabo C, Ohshima H (1997) DNA damage induced by peroxynitrite: subsequent biological effects. Nitric Oxide 1:373-385.

Virag L, Szabo C (1999) Inhibition of poly(ADP-ribose) synthetase (PARS) and protection against peroxynitrite-induced cytotoxicity by zinc chelation. Br J Pharmacol 126:769-777.

Virag L, Scott GS, Cuzzocrea S, Marmer D, Salzman AL, Szabo C (1998) Peroxynitrite-induced thymocyte apoptosis: the role of caspases and poly (ADP-ribose) synthetase (PARS) activation. Immunology 94:345-355.

Wang GJ, Chung HJ, Schnuer J, Pratt K, Zable AC, Kavanaugh MP, Rosenberg PA (1998) High affinity glutamate transport in rat cortical neurons in culture. Mol Pharmacol 53:88-96.

Wang H, Joseph JA (2000) Mechanisms of hydrogen peroxide-induced calcium dysregulation in PC12 cells. Free Radic Biol Med 28:1222-1231.

Wang H, Li J, Follett PL, Zhang Y, Cotanche DA, Jensen FE, Volpe JJ, Rosenberg PA (2004) 12-Lipoxygenase plays a key role in cell death caused by glutathione depletion and arachidonic acid in rat oligodendrocytes. Eur J Neurosci 20:2049-2058.

Weiss JH, Sensi SL, Koh JY (2000) Zn(2+): a novel ionic mediator of neural injury in brain disease. Trends Pharmacol Sci 21:395-401.

Yuan J, Lipinski M, Degterev A (2003) Diversity in the mechanisms of neuronal cell death. Neuron 40:401-413.

Zhang Y, Rosenberg PA (2002) The essential nutrient pyrroloquinoline quinone may act as a neuroprotectant by suppressing peroxynitrite formation. Eur J Neurosci 16:1015-1024.

Zhang Y, Rosenberg PA (2004) Caspase-1 and poly (ADP-ribose) polymerase inhibitors may protect against peroxynitrite-induced neurotoxicity independent of their enzyme inhibitor activity. Eur J Neurosci 20:17271736. 